\title{
BIALGEBRA AND GEOMETRY OF PLANE QUARTICS*
}

\author{
MARAT GIZATULLIN ${ }^{\dagger}$
}

Abstract. Weak semisimplicity of the bialgebra of ternary quartic forms is proved.

In fact there being no direct method of proceeding from the invariant to the geometric meaning ...

If it be not obtained we should console ourselves with the reflexion that the uninterpreted forms are of little geometrical interest in the present state of knowledge;

Besides if we regard the algebra as being merely helpful to geometry in the analytical formulations of results, it does not follow that everything in the algebra need be taken seriously from the geometrical point of view.

(From No. 229 of "The algebra of Invariants" by J. H. Grace and A. Young, Cambridge, 1903)

\section{First definitions and introduction.}

(1.0) Conventions. In the article, the ground field $K$ is always of characteristic zero and algebraically closed.

Let $W$ be a finite-dimensional $K$-vector space, $W^{*}$ be the dual space of $W$. Sometimes, elements of $W$ (denoted as $a, b, c \ldots$ ) will be called linear forms, elements of $W^{*}$ (denoted as $p, q, r \ldots$ ) will be called vectors. Therefore, elements of $\mathbb{P}(W)$ are hyperplanes (or lines, if $W$ is three-dimensional), elements of $\mathbb{P}\left(W^{*}\right)$ are points. Also, we will use geometric term pencil for two-dimensional subspaces of $W$ (or $W^{*}$ ). If $\{a, b\}$ is a basis of a pencil $P$, then we will denote the pencil $P$ by $(\lambda a+\mu b)$ or by $(K a+K b)$. Let

$$
\begin{aligned}
& W \times W^{*} \rightarrow K,(a, p) \mapsto<a, p>\in K, \\
& W^{*} \times W \rightarrow K,(p, a) \mapsto<p, a>\in K
\end{aligned}
$$

be two natural pairings denoted with the same symbol $<,>$.

We will say that two bases $\left\{e_{1}, \ldots, e_{n}\right\} \subset W,\left\{f_{1}, \ldots, f_{n}\right\} \subset W^{*}$ are projectively dual, if $\left\langle e_{i}, f_{j}\right\rangle=0$ for $i \neq j,<e_{i}, f_{i}>\neq 0$. Here, projective point of view means that we do not try to normalize elements of the bases and consider them up to proportionality.

(1.1) Definition. Bialgebra. Bimultiplication law on $W$ (defining a bialgebra structure on W) consists of two bilinear maps (both of which will be denoted by square brackets)

$$
\begin{array}{ll}
W \times W \rightarrow W^{*}, & (a, b) \mapsto[a, b] \in W^{*}, \\
W^{*} \times W^{*} \rightarrow W, & (p, q) \mapsto[p, q] \in W .
\end{array}
$$

The number $\operatorname{dim}(W)$ is dimension (or rank ) of the bialgebra $(W,[]$,$) .$

The number $(\operatorname{dim}(W)-1)$ is projective dimension of the bialgebra.

* Received April 7, 2000; accepted for publication May 17, 2000.

$\dagger$ Max-Planck-Institut for Math., Vivatsgasse 7, 53111 Bonn, Germany and Universidad Técnica Federico Santa María, Avenida España, 1680, Casilla 110-V, Valparaíso, Chile (gizatull@mpimbonn.mpg.de, mgizatul@mat.utfsm.cl). 
Bialgebra $(W,[]$,$) is commutative if$

$$
[a, b]=[b, a], \quad[p, q]=[q, p] \quad \text { for any } \quad a, b \in W, \quad p, q \in W^{*} .
$$

(1.2) Notation. Reciprocal of $x$. For $x \in W \cup W^{*}$, we denote $\widehat{x}=[x, x] / 2$, the element $\widehat{x}$ is the reciprocal of $x$.

Sometimes, we (together with G. Salmon [25], No. 292) will use notation $\sigma(x)$ (instead of $\widehat{x}$ ) for elements $x$ of a bialgebra defined (according to (3.2) and (8.1)) on the space $S^{4}\left(K^{3}\right)$.

We have two (quadratic) polynomial maps ^ ${ }^{\wedge} W \rightarrow W^{*}, \wedge: W^{*} \rightarrow W$, and their composites $\widehat{\wedge}: W \rightarrow W, \widehat{\approx}: W^{*} \rightarrow W^{*}$, also corresponding projectivizations (which are rational maps), for example, the map

$$
\mathbb{P}(\widehat{\wedge}): \mathbb{P}(W)--\rightarrow \mathbb{P}(W) .
$$

(1.3) Definition. Subalgebra and ideal. If $(W,[]$,$) is a bialgebra, then its$ subalgebra is a pair $(U, V)$ of vector subspaces $U \subset W, V \subset W^{*}$ such that

(i) the induced pairing $U \times V \rightarrow K$ is nondegenerate

(therefore $\operatorname{dim} U=\operatorname{dim} V$, this number is said to be the subalgebra dimension, moreover, one can identify $V$ with $U^{*}$ ),

(ii) $[U, U] \subset V, \quad[V, V] \subset U$.

Ideal in $(W,[]$,$) is a subalgebra (U, V)$ such that $[U, W] \subset V,\left[V, W^{*}\right] \subset U$.

(1.4) Definition. Projective idempotent. An element $x \in W \cup W^{*}$ is an idempotent (more precisely, a projective idempotent) if

$$
<x,[x, x]>\neq 0 \text {, and }[[x, x],[x, x]]=k x
$$

for some nonzero $k \in K$, that is $\widehat{\widehat{x}}=k x$.

(1.5) Remark. Geometrically, if $x$ is an idempotent, then the corresponding point $a \in \mathbb{P}(W) \cup \mathbb{P}\left(W^{*}\right)$ belongs to the domain of definition of the rational map $\mathbb{P}(\widehat{\wedge})$, and this point is a fixed point of the map.

Algebraically, $a \in W$ is an idempotent if and only if $(K a, K[a, a])$ is a onedimensional (projectively zero-dimensional) subalgebra of $(W,[]$,$) , therefore, there$ exists a correspondence between projective idempotents and one-dimensional subalgebras.

With the point of view of the generalization given by Definition (1.6) below, ordinary idempotent is an idempotent of level zero.

(1.6) Definition. Idempotents of level $m$. An element $x \in W \cup W^{*}$ of bialgebra $(W,[]$,$) is an idempotent of level m$, if there exists a subalgebra $(U, V) \subset\left(W, W^{*}\right)$ of dimension $\leq m+1$ containing $x$.

Weak idempotent is an idempotent of level 1 , projectively onedimensional subalgebras will be called idempotent pencils. Thus, idempotent pencil defines a line in $\mathbb{P}(W)$, the line is invariant with respect to the map $\mathbb{P}(\widehat{ })$ (also, a line in $\mathbb{P}\left(W^{*}\right)$ with a similar property, both the lines are reciprocal images by $\mathbb{P}(\nearrow)$.

(1.7) Definition. Semisimplicity of level $m$. An $n$-dimensional commutative bialgebra $(W,[]$,$) is called m$-semisimple (or semisimple of level $m$ ), if there are $n$ subalgebras $\left(U_{1}, V_{1}\right), \ldots,\left(U_{n}, V_{n}\right)$ of $(W,[]$,$) and two subsets$

$$
\left\{e_{1}, \ldots, e_{n}\right\} \subset W, \quad\left\{f_{1}, \ldots, f_{n}\right\} \subset W^{*}
$$


satisfying the following conditions:

(i) $\operatorname{dim} U_{i} \leq m+1, \quad \operatorname{dim} V_{i} \leq m+1(i \in\{1,2, \ldots, n\})$,

(ii) $e_{i} \in U_{i}, f_{i} \in V_{i}$ (therefore $e_{i}, f_{i}$ are idempotents of level $m$ ),

(iii) elements $\widehat{e}_{1}, \ldots, \widehat{e}_{n}$ constitute a base for $W^{*}$, and this base is dual of the base $\left\{e_{i}\right\}$,

(iv) elements $\widehat{f}_{1}, \ldots, \widehat{f}_{n}$ constitute a base of $W$, and it is dual of the base $\left\{f_{i}\right\}$.

Also, 1-semisimple bialgebra will be called weakly semisimple.

0 -semisimple bialgebra is called semisimple, more precisely, a commutative bialgebra $(W,[]$,$) is said to be semisimple, if there exists a base \left\{e_{1}, \ldots, e_{n}\right\}(n=\operatorname{dim} W)$ for $W$ such that $e_{i}$ are idempotents, elements $\widehat{e}_{1}, \ldots, \widehat{e}_{n}$ constitute a base for $W^{*}$ and these bases are mutually dual. For example, the bialgebra of binary quartics defined in (4.2) is semisimple. We will publish a proof of this result in other place.

It is known (see Theorem (7.11)) that the bialgebra of ternary quadratic forms (with respect to the natural bimultiplication described in no. 6 below) is semisimple. Actually, it was established by Francesco Gerbaldi in 1882 (see [11], [12], [13], [14], also comments in [9], [16], [19], [34]), he has constructed the so called six mutually apolar conics. The conics were used for a description of Valentiner's subgroup [28] $G_{360}$ of $\operatorname{Aut}\left(\mathbb{P}^{2}\right)$ : as an abstract group, $G_{360}$ is isomorphic to the alternating group $A_{6}$ of even permutations of six symbols, Gerbaldi demonstrated that one can take the conics as the symbols, that is Valentiner's group interchanges the conics, see [34] for some modern comments. Moreover, the conics were used for a construction of a point-line configuration studied by Gerbaldi (loc. cit.). The set of intersection points of distinct Gerbaldi's conics consists of 60 points. From pure geometric consideration, without use of Gerbaldi's conics, the configuration was constructed by W. Burnside [3], whose attention was concentrated on 45 points of the dual plane (see also E. Steinitz' and A. Wiman's surveys [27], [33] and modern paper [34] by S. Grass). We will call it as the Gerbaldi-Burnside configuration.

For the bialgebra of ternary forms of the next even degree, that is for the bialgebra of ternary quartics described in (8.1), the question about 0 -semisimplicity is open, but we will prove a weak assertion.

(1.9) Theorem. The bialgebra of ternary quartics is weakly semisimple.

In the proof of the theorem, we shall construct fifteen quartic idempotent pencils $\left(U_{i}, V_{i}\right) \quad(i=1,2, \ldots, 15)$, where $U_{i}$ is the cogredient part, defining a pencil of curves in $\mathbb{P}(W), V_{i}$ is the contragredient part, defining a pencil of curves in $\mathbb{P}\left(W^{*}\right)$. Each of the parts is projectively equivalent to the so-called Wiman quartic pencil. Such a pencil was considered by A. Wiman [31], [32], and also by E. Ciani, W. L. Edge [6], I. Dolgachev and V. Kanev [5], R. E. Rodríguez and V. González-Aguilera [23]. Each of the fifteen pencils $U_{i}$ contains two Klein's quartics, one Fermat's quartic, a Capolari quartic, a Clebsch quartic and a Bernoulli lemniscate. Each pair $\left(U_{i}, V_{i}\right)$ is projectively isomorphic to Wiman quartic subalgebra from our Definition (10.11) with $2 m=4$ and from (11.9-12).

(1.10) Theorem. The union of all base loci of the fifteen Wiman quartic pencils coincides with the set of 60 points of the Gerbaldi-Burnside configuration.

In the quartic bialgebra, another weakened property of semisimplicity holds.

(1.11) Theorem. In the bialgebra of ternary quartics, one can find fifteen sixdimensional and SEMISIMPLE subalgebras with the properties indicated in definition (1.7) (where $m=6$ is taken of course).

An example of the quartic subalgebra of Theorem (1.11) was considered by G. 
Salmon in his "Treatise on higher plane curves" [25], no. 298, therefore we will call such a subalgebra as Salmon's subalgebra.

Our proofs and constructions are inscribed in a framework of some general considerations from the bialgebras theory and the theory of co(contra)variants of ternary forms. Also we give some illustrative examples connected with the invariant theory of binary forms or with ternary forms of arbitrary degree, but in this text, the cases of binary forms or ternary forms of the degree distinct from 2 and 4 are used for an illustration of the notion of bimultiplication only, that is we do not consider a structure or semisimplicity of corresponding bialgebras, although, in (13.7), we give an example of a four-dimensional semisimple subalgebra of the bialgebra of ternary sextics, this bialgebra contains a set of remarkable sextics (for example, a couple of idempotent Wiman's sextics $C, C^{\prime}$ with the property $\operatorname{Aut}(C)=\operatorname{Aut}\left(\mathbb{P}^{2}, C\right)=G_{360}$, Wiman's sextic $W$ with $\operatorname{Aut}(W)=\operatorname{Bir}\left(\mathbb{P}^{2}, W\right)=G_{120}$, and a couple of rational ten-nodal sextics). A short description of bialgebras arising from semisimple Lie algebras was added by the revision of the text in (5.10).

Acknowledgements. The search for the description of the fifteen idempotent quartic pencils was begun in 1999 during my stay at Max-Planck-Institut für Mathematik. I am indebted to the administration of MPIM for having made possible this visit, I would like to express my gratitude to the Institute for hospitality, financial support and excellent work conditions.

The main theorem (1.9) was a subject of my talk at Yu. I. Manin's MPIM "Seminar on Algebra, Geometry and Physics" (29th of February, 2000). The term "potential" used in the bialgebra context below is borrowed from his MPIM-lectures "Moduli, Motives, Mirrors and other Marvels". I would like to express to him my gratitude.

I would like to thank Dale Husemöller for corrections of my English text.

After revising the initial text, I would like to thank the referee for exact remarks, corrections and improvements. Paper [34] was indicated by the referee.

\section{Symmetric and skew-symmetric bialgebras.}

(2.1) Definition. Symmetric and skew-symmetric bialgebras, their potentials. The bimultiplication law [,] is symmetric (resp. skew) if both the trilinear functions $\phi(a, b, c)=<[a, b], c>, \psi(p, q, r)=<[p, q], r>$

$$
\left(\phi: W \times W \times W \rightarrow K, \quad \psi: W^{*} \times W^{*} \times W^{*} \rightarrow K\right)
$$

are symmetric (resp. alternative). Sometimes we will write $\langle a . b . c\rangle$ instead of $\phi(a, b, c)$. We will be concerned with symmetric or skew-symmetric bimultiplications only. In both these cases, the pair $(\phi, \psi)$ of these trilinear functions is called bipotential for the bimultiplication, components of the bipotential are potentials. Of course, the pair of these functions is the same as a pair of tensors $\left(t, t^{*}\right)$, where $t \in\left(W^{*}\right)^{\otimes 3}$, $t^{*} \in(W)^{\otimes 3}$. Both of the tensors are symmetric (resp. skew-symmetric) for symmetric (resp. skew) bialgebra. For the case of symmetric bialgebra, the homogeneous functions $i(a)=\phi(a, a, a), \quad i^{*}(p)=\psi(p, p, p)$ are called potentials also, because the latter pair of functions determines bipotential for symmetric bialgebra.

It is evident that symmetric bialgebra is commutative: $[a, b]=[b, a], \quad[p, q]=$ $[q, p]$, skew bialgebra is anti-commutative: $[a, b]=-[b, a], \quad[p, q]=-[q, p]$. We will consider only those bialgebra properties which do not depend on the change of a bimultiplication for proportional bimultiplication, and sometimes we will change the bipotential $(\phi, \psi)$ for $(k \phi, l \psi)$, where $k, l$ are nonzero constants. 
(2.2) Remark. The notions of idempotent, semisimplicity or $m$-semisimplicity (definitions(1.4), (1.7), (1.8)) do not have a sense for skew bialgebras, but skew bialgebras will be used for constructions of symmetric bialgebras in (3.2-3), (4,2-4).

Note that for symmetric or skew-symmetric bialgebra, the bipotential determines the bimultiplication: element $p=[a, b] \in W^{*}$ is defined by the condition $\langle p, x\rangle=$ $\phi(a, b, x)$ for all $x \in W$, similarly, element $a=[p, q] \in W$ is defined by the condition $<a, y>=\psi(p, q, y)$ for all $y \in W^{*}$.

Our use of word "potential" can be explained by gradient-like character of formulas for bimultiplications or reciprocals, see $(4,4), 6,(8,1)$.

(2.3) Definition. Special bialgebra. A symmetric or skew-symmetric bialgebra $(W,[]$,$) is said to be special, if there is a pair of projectively dual bases such that the$ linear isomorphism $W \rightarrow W^{*}$, defined with the help of the bases induces a coincidence (up to proportionality) of the bipotential components, that is functions

$$
\phi\left(\sum x_{i} e_{i}, \sum y_{i} e_{i}, \sum z_{i} e_{i}\right), \psi\left(\sum x_{i} f_{i}, \sum y_{i} f_{i}, \sum z_{i} f_{i}\right)
$$

are proportional.

(2.4) Definition. Projectively equivariant bimultiplication.

(1) Remark. Assume that a group $G$ acts linearly on $W$. We say that a bialgebra structure on $W$ is projectively $G$-equivariant (or $\mathbb{P}(G)$-equivariant), if for some multiplicative character $\chi$ of $G$, for any $g \in G$ and for any $a, b \in W$, we have $[g(a), g(b)]=\chi(g)\left(g^{t}\right)^{-1}([a, b])$, where $g^{t}: W^{*} \rightarrow W^{*}$ is the contragredient (transpose) map.

(2) If a bimultiplication on $W$ is projectively $\mathrm{GL}(W)$-equivariant, then we say that it is projectively equivariant or $\operatorname{PGL}(W)$-equivariant.

In the case of a symmetric projectively equivariant bimultiplication, the potential components $\phi$ and $\psi$ are simultaneous invariants of the argument triple:

$$
\phi(g(a), g(b), g(c))=\chi_{1}(g) \phi(a, b, c), \quad \psi(g(p), g(q), g(r))=\chi_{2}(g) \phi(p, q, r)
$$

for some characters $\chi_{1}, \chi_{2}$.

(2.5) Notation. For special symmetric bimultiplication and $x \in W \cup W^{*}$, we will denote $I(x)=<x,[x, x]>/ 3$. In this case, we will call this cubic homogeneous function as potential also.

Note that for projectively equivariant bimultiplication, the function $I(x)$ is an invariant. The reciprocal $\widehat{x}$ of $x$ is a contravariant of $x$.

(2.6) Remark. For any pair of elements $a \in W, b \in W$ of a symmetric bialgebra $(W,[]$,$) , and for any constants x \in K, y \in K$, the following identity

$$
\widehat{x a+y} b=x^{2} \widehat{a}+x y[a, b]+y^{2} \widehat{b}
$$

holds.

\section{Extension of bimultiplication to symmetric powers.}

(3.1) Notation. We will denote by $\langle,>$ the extension of the duality pairing $<,>$ to the symmetric powers $S^{n}(W)$, more precisely,

$$
<., .>: S^{n}(W) \times S^{n}\left(W^{*}\right) \rightarrow K
$$


is such that $\left.\left\langle a^{n}, p^{n}\right\rangle=<a, p\right\rangle^{n}$, and therefore

$$
<a^{n}, p_{1} p_{2} \ldots p_{n}>=<a, p_{1}><a, p_{2}>\ldots<a, p_{n}>\text {. }
$$

(3.2) Theorem. Let $(W,[]$,$) be a bialgebra with either skew-symmetric or$ symmetric PGL $(W)$-equivariant bimultiplication. Then for any natural $m$, there exists a unique $\operatorname{PGL}(W)$-equivariant bimultiplication on $S^{m}(W)$ such that

$$
\left[a^{m}, b^{m}\right]=[a, b]^{m}, \quad\left[p^{m}, q^{m}\right]=[p, q]^{m},
$$

where $a, b \in W, \quad p, q \in W^{*}$.

If the initial bimultiplication is symmetric (resp. special), then new bimultiplications are also symmetric (resp. special).

If the initial bimultiplication is skew, then the bimultiplication on $S^{m}(W)$ is skew by odd $m$ and symmetric by even $m$.

Proof. We write only a sketch of a proof. A complete proof of a general theorem including an analog of theorem (3.3) for the case of $m$-algebras will be published in another place.

An informal explanation (with the point of view of invariantists of XIX-th century) is based on the symbolic method: if $\phi(a, b, c)$ is a potential for the bimultiplication in $(W,[]$,$) , and elements f, g, h \in S^{m}(W)$ are written symbolically as $f=a^{m}, g=b^{m}, h=c^{m}$, then the potential $\phi_{m}$ for the bimultiplication on $S^{m}(W)$ is defined symbolically by $\phi_{m}(f, g, h)=(\phi(a, b, c))^{m}$.

A bit more serious explanation is the following. We can define a bimultiplication with the help of the formulas in (3.2), but linear relations between powers of linear forms might spoil such a definition. Therefore we need the following lemma.

Lemma. Let $W, V$ be vector spaces,

$$
W \times W \rightarrow V, \quad(a, b) \mapsto[a, b]
$$

be a bilinear map. If the relation $\sum a_{i}^{m}=0$ holds in $S^{m}(W)$, then for any $b \in W$, also the relation $\sum\left[a_{i}, b\right]^{m}=0$ holds in $S^{m}(V)$.

Proof. The lemma is obvious after writing all the relations with the help of coordinates.

This proves the lemma and the theorem.

One can generalize Theorem (3.2) by the following way.

(3.3) Theorem. Let $k$ be a natural number. Suppose that it is given a PGL $(W)$ equivariant (with respect to the representation in $k$-th symmetric power of $W$ ) bimultiplication

$$
\text { [., .] : } S^{k}(W) \times S^{k}(W) \rightarrow S^{k}\left(W^{*}\right), \quad[., .]: S^{k}\left(W^{*}\right) \times S^{k}\left(W^{*}\right) \rightarrow S^{k}(W) .
$$

Suppose that this bimultiplication is either skew-symmetric or symmetric. Then for any natural $m$, there exists a unique $\operatorname{PGL}(W)$-equivariant bimultiplication

$$
[., .]: S^{m k}(W) \times S^{m k}(W) \rightarrow S^{m k}\left(W^{*}\right), \quad[., .]: S^{m k}\left(W^{*}\right) \times S^{m k}\left(W^{*}\right) \rightarrow S^{m k}(W),
$$

such that

$$
\left[a^{k m}, b^{k m}\right]=[a, b]^{k m}, \quad\left[p^{k m}, q^{k m}\right]=[p, q]^{k m},
$$

where $a, b \in W, \quad p, q \in W^{*}$.

If the initial bimultiplication is symmetric (resp. special), then new bimultiplications are also symmetric (resp. special). 
If the initial bimultiplication is skew, then the bimultiplication on $S^{m k}(W)$ is skew by odd $m$ and symmetric by even $m$.

The proof is almost the same as for Theorem (3.2), therefore we omit it. Moreover, the theorem (3.3) will be used for consideration of an inessential example of binary forms.

(3.4) Remark. Existence of an extension of a given bimultiplication to other tensors is a problem. For example, if $\operatorname{dim} W=3$ and we try to extend (by a $\operatorname{PGL}(W)$ equivariant way ) to $W \otimes W$ the symmetric bimultiplication on $S^{2}(W)$ (such a bimultiplication exists in virtue of Theorem (3.2) and the basic example below), then we will get nothing, because a non-zero cubic invariant of bilinear forms is absent (according to M. Pash [22]).

4. The case of binary forms. The space $V_{m}=S^{m}(V)(\operatorname{dim} V=2)$ of binary forms of degree $m$

$$
\left(V_{m}=\left\{a \mid a=\sum_{i=0}^{m} a_{i}\left(\begin{array}{c}
m \\
i
\end{array}\right) x_{0}^{i} x_{1}^{m-i}\right\}\right)
$$

and its dual $V_{m}^{*}$

$$
\left(V_{m}^{*}=\left\{p \mid p=\sum_{i=0}^{m} p_{i}\left(\begin{array}{c}
m \\
i
\end{array}\right) u_{0}^{i} u_{1}^{m-i}\right\}\right)
$$

can be projectively (and by PGL(2)-equivariant way) identified with the help of substitution

$$
x_{0}=-u_{1}, x_{1}=u_{0},
$$

but we will distinguish both the spaces. There are natural (and mutually contragredient) actions of GL $(V)$ on both the spaces. First, let us consider the three-dimensional space $V_{2}$ of binary quadratic forms. There is a natural special PGL(V)-equivariant skew bimultiplication

$$
V_{2} \times V_{2} \rightarrow V_{2}^{*}, \quad V_{2}^{*} \times V_{2}^{*} \rightarrow V_{2}
$$

it is the Jacobian (where the above mentioned change of variables (4.1) is done):

$$
[a, b]=2\left|\begin{array}{cc}
a_{1} u_{0}-a_{0} u_{1} & a_{2} u_{0}-a_{1} u_{1} \\
b_{1} u_{0}-b_{0} u_{1} & b_{2} u_{0}-b_{1} u_{1}
\end{array}\right|
$$

The potential is the following simultaneous invariant of three quadratic forms

$$
\phi(a, b, c)=\left|\begin{array}{lll}
a_{0} & a_{1} & a_{2} \\
b_{0} & b_{1} & b_{2} \\
c_{0} & c_{1} & c_{2}
\end{array}\right|
$$

Note that this bialgebra is projectively isomorphic to the three-dimensional bialgebra of theorem (5.1) below, because the potentials are the same. According to theorem (3.3), we get a structure of a skew bialgebra on $V_{2 m}$, where $m$ is odd, and a structure of a symmetric bialgebra on $V_{4 m}$.

One can present a description of the bimultiplication for $V_{2 m}$ in terms of the so called symbolic method:

$$
\text { if } a=a_{x}^{2 m}, \quad b=b_{x}^{2 m}, \quad c=(a, b)^{m} a_{x}^{m} b_{x}^{m}, \quad \text { then }[a, b]=c\left(-u_{1}, u_{0}\right) .
$$


(4.2) Example. Binary quartics. For the space $V_{4}$ of binary forms of degree 4 , the potential of the special bimultiplication is the following Hankel determinant

$$
I(a)=\left|\begin{array}{lll}
a_{0} & a_{1} & a_{2} \\
a_{1} & a_{2} & a_{3} \\
a_{2} & a_{3} & a_{4}
\end{array}\right|,
$$

the reciprocal quartic $\widehat{a}$ is transformed (with the help of the substitution (4.1)) the Hesse covariant (that is $\widehat{a}$ is the Hesse reciprocant according to the terminology of J. Sylvester). Thus, $\widehat{a}$ is equal to

$$
\begin{gathered}
6\left(a_{4} a_{2}-a_{3}^{2}\right) u_{0}^{4} \\
+4 \cdot 3\left(a_{2} a_{3}-a_{4} a_{1}\right) u_{0}^{3} u_{1} \\
+6\left(a_{0} a_{4}+2 a_{1} a_{3}-3 a_{2}^{2}\right) u_{0}^{2} u_{1}^{2} \\
+4 \cdot 3\left(a_{1} a_{2}-a_{0} a_{3}\right) u_{0} u_{1}^{3} \\
+6\left(a_{0} a_{2}-a_{1}^{2}\right) u_{1}^{4} .
\end{gathered}
$$

The symmetric bialgebra of binary quartics is semisimple. The set of nonzero nilpotent elements coincides with the set of not semi-stable elements ( $N u l l$-formen) in the sense of Hilbert-Mumford. A description of these facts will be published in other place.

(4.3) Example. Binary sextics. For two binary sextics $a, b \in V_{6}$, their product $p=[a, b]$ has the following coefficients.

$$
\begin{aligned}
& p_{0}=a_{3} b_{6}-3 a_{4} b_{5}+3 a_{5} b_{3}-a_{6} b_{3}, \\
& p_{1}=\frac{1}{2}\left(-a_{2} b_{6}+2 a_{3} b_{5}-2 a_{5} b_{3}+a_{6} b_{2}\right), \\
& p_{2}=\frac{1}{5}\left(a_{1} b_{6}-5 a_{3} b_{4}+5 a_{4} b_{3}-a_{6} b_{1}\right), \\
& p_{3}=\frac{1}{20}\left(-a_{0} b_{6}-6 a_{1} b_{5}+15 a_{2} b_{4}-15 a_{4} b_{2}+6 a_{5} b_{1}+a_{6} b_{0}\right), \\
& p_{4}=\frac{1}{5}\left(a_{0} b_{5}-5 a_{2} b_{3}+5 a_{3} b_{2}-a_{5} b_{0}\right) \\
& p_{5}=\frac{1}{2}\left(-a_{0} b_{4}+2 a_{1} b_{3}-2 a_{3} b_{1}+a_{4} b_{0}\right), \\
& p_{6}=a_{0} b_{3}-3 a_{1} b_{2}+3 a_{2} b_{1}-a_{3} b_{0} .
\end{aligned}
$$

(4.4) Example. Binary octavics. For a description of a symmetric special bimultiplication on $V_{8}$, it is enough to indicate $\widehat{a}$, because formulas for $[a, b]$ are derivable by the process of bilinearization. If $a$ is a binary octavic, then there exists an octavic covariant $i=(a, a)_{4}$ (according to notations of von Gall in [10]; von Gall refers to P. Gordan's lectures [15] of 1875$)$. Then $\widehat{a}=i\left(-u_{1}, u_{0}\right)$, the coefficients $\widehat{a}_{k}$ of $\widehat{a}$ are written down below.

$$
\begin{gathered}
\widehat{a}_{0}=a_{4} a_{8}-4 a_{5} a_{7}+3 a_{6}^{2}=\frac{\partial I(a)}{\partial a_{0}} \\
\widehat{a}_{1}=\frac{1}{2}\left(-a_{3} a_{8}+3 a_{4} a_{7}-2 a_{5} a_{6}\right)=\frac{1}{8} \frac{\partial I(a)}{\partial a_{1}} \\
\widehat{a}_{2}=\frac{1}{14}\left(3 a_{2} a_{8}-4 a_{3} a_{7}-11 a_{4} a_{6}+12 a_{5}^{2}\right)=\frac{1}{28} \frac{\partial I(a)}{\partial a_{2}}
\end{gathered}
$$




$$
\begin{gathered}
\widehat{a}_{3}=\frac{1}{14}\left(-a_{1} a_{8}-2 a_{2} a_{7}+12 a_{3} a_{6}-9 a_{4} a_{5}\right)=\frac{1}{56} \frac{\partial I(a)}{\partial a_{3}} \\
\widehat{a}_{4}=\frac{1}{70}\left(a_{0} a_{8}+12 a_{1} a_{7}-22 a_{2} a_{6}-36 a_{3} a_{5}+45 a_{4}^{2}\right)=\frac{1}{70} \frac{\partial I(a)}{\partial a_{4}} \\
\widehat{a}_{5}=\frac{1}{14}\left(-a_{0} a_{7}-2 a_{1} a_{6}+12 a_{2} a_{5}-9 a_{3} a_{4}\right)=\frac{1}{56} \frac{\partial I(a)}{\partial a_{5}} \\
\widehat{a}_{6}=\frac{1}{14}\left(3 a_{0} a_{6}-4 a_{1} a_{5}-11 a_{2} a_{4}+12 a_{3}^{2}\right)=\frac{1}{28} \frac{\partial I(a)}{\partial a_{4}} \\
\widehat{a}_{7}=\frac{1}{2}\left(-a_{0} a_{5}+3 a_{1} a_{4}-2 a_{2} a_{3}\right)=\frac{1}{8} \frac{\partial I(a)}{\partial a_{7}} \\
\widehat{a}_{8}=a_{0} a_{4}-4 a_{1} a_{3}+3 a_{2}^{2}=\frac{\partial I(a)}{\partial a_{8}}
\end{gathered}
$$

where the potential $I(a)$ for the bialgebra $V_{8}$ of binary octavics coincides with the invariant $J_{3}$ of Gordan's list of 1875 (there is a reference of von Gall [10] to the list). The explicit formula for the potential is

$$
\begin{gathered}
I(a)=a_{0} a_{4} a_{8}+15 a_{4}^{3}+12 a_{1} a_{4} a_{7}-22 a_{2} a_{4} a_{6}-36 a_{3} a_{4} a_{5} \\
-4\left(a_{0} a_{5} a_{7}+a_{1} a_{3} a_{8}\right)-8\left(a_{1} a_{5} a_{6}+a_{2} a_{3} a_{7}\right) \\
+24\left(a_{2} a_{5}^{2}+a_{6} a_{3}^{2}\right)+3\left(a_{0} a_{6}^{2}+a_{8} a_{2}^{2}\right) .
\end{gathered}
$$

\section{The basic example of a threedimensional skew bialgebra.}

(5.1) Theorem. There exists a (projectively) unique skew-symmetric special three-dimensional bialgebra $(W,[]$,$) with nonzero and PGL (W)$-equivariant bimultiplication.

Proof. For three-dimensional vector space, there exists a unique (up to proportionality) trilinear alternating function.

(5.2) Remark. In some sense, this bimultiplication is the same as in the above case of binary quadratic forms, but we will distinguish the cases.

Let us fix a threedimensional bialgebra $(W,[]$,$) mentioned in Theorem (5.1) with$ a trilinear potential $\langle a . b . c\rangle$.

Identities $(5,3-8)$ for this threedimensional bialgebra and its symmetric powers are written below, one can verify them with the help of the coordinate description (5.9). Moreover, when we will write down identities for linear forms $a, b \ldots \in W$, then we will omit the parallel identities for vectors $p, q, . . \in W^{*}$, if the written identities contain linear forms only.

$$
\begin{aligned}
& a<b . c . d>-b<c . d . a>+c<d . a . b>-d<a . b . c>=0 \\
& {[[a, b],[c, d]]+[[a, c],[d, b]]+[[a, d],[b, c]]=2 a<b . c . d>}
\end{aligned}
$$

where $a, b, c, d \in W$. Similar identities hold for elements of $W^{*}$. In $S^{2}(W)$, for some squares of elements of $W$, the following identity holds.

$$
\begin{aligned}
& {[[a, b],[c, d]]^{2}+[[a, c],[b, d]]^{2}+[[a, d],[b, c]]^{2} } \\
= & \langle b . c . d\rangle^{2} a^{2}+\langle a . c . d\rangle^{2} b^{2}+\langle a . b . d\rangle^{2} c^{2}+\langle a . b . c\rangle^{2} d^{2} .
\end{aligned}
$$


In $S^{4}(W)$, we have

$$
\begin{aligned}
& {[[a, b],[c, d]]^{4}+[[a, c],[b, d]]^{4}+[[a, d],[b, c]]^{4} } \\
= & <\text { b.c.d }>^{4} a^{4}+<a . c . d>^{4} b^{4}+<a . b . d>^{4} c^{4}+<a . b . c>^{4} d^{4} \\
& +12<\text { b.c.d }><\text { a.c.d }><\text { a.b.d }><\text { a.b.c }>\text { abcd. }
\end{aligned}
$$

Further, in $S^{n+k}(W)$, we have

$$
\left(\begin{array}{c}
n+k \\
k
\end{array}\right)\left[a^{n} b^{k}, b^{n} c^{k}\right]=[a, b]^{n}[b, c]^{k} .
$$

Also we will use the following simple formula which is a corollary of (5.4).

$$
[[a, b],[a, c]]=\langle a . b . c\rangle \cdot a .
$$

(5.9) The coordinate description of the basic example. Let

$$
\left\{x_{0}, x_{1}, x_{2}\right\} \subset W, \quad\left\{u_{0}, u_{1}, u_{2}\right\} \subset W^{*}
$$

be two reciprocal bases, $\left\langle u_{i}, x_{j}\right\rangle=\left\langle x_{j}, u_{i}\right\rangle=\delta_{i j}$. The quantities $x_{0}, x_{1}, x_{2}$ are the so called cogredient variables, $u_{0}, u_{1}, u_{2}$ are contragredient ones. If

$$
a=a_{0} x_{0}+a_{1} x_{1}+a_{2} x_{2} \in W, \quad p=p_{0} u_{0}+p_{1} u_{1}+p_{2} u_{2} \in W^{*},
$$

then $\langle a, p\rangle=a_{0} p_{0}+p_{1} u_{1}+p_{2} u_{2}$. We may choose two trilinear potential functions

$$
\phi: W \times W \times W \rightarrow \bigwedge^{3}(W) \cong K, \quad \psi: W^{*} \times W^{*} \times W^{*} \rightarrow \bigwedge^{3}\left(W^{*}\right) \cong K
$$

as determinants: if

$$
\begin{aligned}
& a=a_{0} x_{0}+a_{1} x_{1}+a_{2} x_{2}, b=b_{0} x_{0}+b_{1} x_{1}+b_{2} x_{2}, c=c_{0} x_{0}+c_{1} x_{1}+c_{2} x_{2}, \\
& p=p_{0} u_{0}+p_{1} u_{1}+p_{2} u_{2}, q=q_{0} u_{0}+q_{1} u_{1}+q_{2} u_{2}, r=r_{0} u_{0}+r_{1} u_{1}+r_{2} u_{2},
\end{aligned}
$$

then

$$
\phi(a, b, c)=<a . b . c>=\left|\begin{array}{lll}
a_{0} & a_{1} & a_{2} \\
b_{0} & b_{1} & b_{2} \\
c_{0} & c_{1} & c_{2}
\end{array}\right|, \quad \psi(p, q, r)=<p . q . r>=\left|\begin{array}{ccc}
p_{0} & p_{1} & p_{2} \\
q_{0} & q_{1} & q_{2} \\
r_{0} & r_{1} & r_{2}
\end{array}\right| .
$$

For two linear forms $a, b$, we have

$$
[a, b]=\left(a_{1} b_{2}-a_{2} b_{1}\right) u_{0}+\left(a_{2} b_{0}-a_{0} b_{2}\right) u_{1}+\left(a_{0} b_{1}-a_{1} b_{0}\right) u_{2} .
$$

(5.10) Example. Example of a bialgebra constructed with the help of a semisimple Lie algebra. It is necessary to explain the place of the basic example in a general picture. Let $W$ be a finite-dimensional semisimple Lie algebra over the ground field $K,\{$,$\} be the bracket in W, M$ be an exact finite-dimensional $W$-module, $a_{M}$ be the operator corresponding to $a \in W$ by the representation. The Killing form of the representation is $(a, b)_{M}=\operatorname{Trace}\left(\left(a_{M}\right) \circ\left(b_{M}\right)\right)$. The Killing form for the regular representation will be denoted by $(a, b)$. Let $f: W \rightarrow W^{*}$ be the isomorphism, corresponding to the Killing form (.,.), that is $\langle f(a), x\rangle=(a, x)$. This is the so-called Killing isomorphism, see 1.2.21,1.5.14 from the book [ 35 ] by J. Dixmier. Then the homomorphisms

$$
\begin{array}{ll}
W \times W \rightarrow W^{*}, & (a, b) \mapsto f(\{a, b\}), \\
W^{*} \times W^{*} \rightarrow W, \quad(p, q) \mapsto\left\{f^{-1}(p), f^{-1}(q)\right\}
\end{array}
$$


define a structure of a special skew bialgebra $(W,[]$,$) .$

If $G$ is an algebraic group such that its Lie algebra is $W$, and $\operatorname{Ad}: G \rightarrow \operatorname{Aut}(W)$ is the adjoint representation, then the bimultiplication [,] is $\mathbb{P}(\mathrm{Ad})$-equivariant.

The alternating property of the potentials

$$
\phi(x, y, z)=<[x, y], z>, \quad \psi(p, q, r)=<[p, q], r>
$$

is a consequence of anticommutativity of Lie's operation $\{$,$\} and of the Jacobi identity,$ but the Jacobi identity is not a consequence of this property, therefore, generally speaking, the notion of bialgebra is wider and more flexible than the notion of Lie algebra. For example, in the contrast with the structure of Lie algebra, one can extend the bialgebra structure to the spaces of symmetric tensors of the initial space.

According to Theorem (3.2), we can extend the bimultiplication to every symmetric power $S^{m}(W)$ of the Lie algebra $W$, and also to every symmetric homogeneous linear subspace $U^{m}(W)$ (defined in 2.4.3 of [35]) of the universal enveloping algebra $U(W)$ of the Lie algebra $W$, because the component is isomorphic to the symmetric power according to [35], 2.4.10.

If $W=\operatorname{sl}(2)$, then we get our basic example.

(5.11) Convention. Further, $W$ will denote the threedimensional space, $(W,[]$, will denote the above described threedimensional bialgebra (the exceptions are Remark (6.6) and general Definition (7.5)) Also, we will use coordinates from (5.9) in further parts of the text.

6. Bialgebra of conics. For quadratic forms $A, B, C \in S^{2}(W)$, the potential $<A . B . C>=<A,[B, C]>$ is the polarized discriminant in the sense that

$$
\operatorname{disc}(A)=\frac{1}{3 !}<A \cdot A \cdot A>=\frac{1}{3}<\widehat{A}, A>.
$$

For two ternary quadratic forms $A \in S^{2}(W), B \in S^{2}(W)$ written as

$$
\begin{aligned}
& A=a_{00} x_{0}^{2}+a_{11} x_{1}^{2}+a_{22} x_{2}^{2}+2 a_{12} x_{1} x_{2}+2 a_{20} x_{2} x_{0}+2 a_{01} x_{0} x_{1}, \\
& B=b_{00} x_{0}^{2}+b_{11} x_{1}^{2}+b_{22} x_{2}^{2}+2 b_{12} x_{1} x_{2}+2 b_{20} x_{2} x_{0}+2 b_{01} x_{0} x_{1},
\end{aligned}
$$

the coefficients of the simultaneous contravariant form

$$
[A, B]=Q\left(u_{0}, u_{1}, u_{2}\right)=\sum_{k, l} q_{k l} u_{k} u_{l}
$$

are

$$
q_{k l}=\sum_{i j} \frac{\partial^{2} \operatorname{disc}(A)}{\partial a_{k l} \partial a_{i j}} b_{i j}=\sum_{i j} \frac{\partial^{2} \operatorname{disc}(B)}{\partial b_{k l} \partial b_{i j}} a_{i j},
$$

or, in more details,

$$
\begin{aligned}
& q_{00}=a_{11} b_{22}+a_{22} b_{11}-2 a_{12} b_{12} \\
& q_{11}=a_{00} b_{22}+a_{22} b_{00}-2 a_{02} b_{02}, \\
& q_{22}=a_{00} b_{11}+a_{11} b_{00}-2 a_{01} b_{01} \\
& q_{12}=a_{10} b_{20}+a_{20} b_{10}-a_{12} b_{00}-a_{00} b_{12} \\
& q_{20}=a_{21} b_{01}+a_{01} b_{21}-a_{20} b_{11}-a_{11} b_{20} \\
& q_{01}=a_{02} b_{12}+a_{12} b_{02}-a_{01} b_{22}-a_{22} b_{01}
\end{aligned}
$$


Especially, coefficients $\widehat{a}_{k l}$ of the dual quadratic form

$$
\widehat{A}\left(u_{0}, u_{1}, u_{2}\right)=\sum_{k, l} \widehat{a}_{k l} u_{k} u_{l}=[A, A] / 2=\left|\begin{array}{cccc}
a_{00} & a_{01} & a_{02} & u_{0} \\
a_{10} & a_{11} & a_{12} & u_{1} \\
a_{20} & a_{21} & a_{22} & u_{2} \\
u_{0} & u_{1} & u_{2} & 0
\end{array}\right|
$$

are the following.

$$
\begin{aligned}
& \widehat{a}_{00}=a_{11} a_{22}-a_{12}^{2}, \\
& \widehat{a}_{11}=a_{00} a_{22}-a_{02}^{2}, \\
& \widehat{a}_{22}=a_{00} a_{11}-a_{01}^{2}, \\
& \widehat{a}_{12}=a_{10} a_{20}-a_{12} a_{00}, \\
& \widehat{a}_{20}=a_{21} a_{01}-a_{20} a_{11}, \\
& \widehat{a}_{01}=a_{02} a_{12}-a_{01} a_{22} .
\end{aligned}
$$

The geometric meaning of the bimultiplication for conics is explained in Salmon's "Conic Sections" [24], No. 335, or in Baker's "Plane Geometry" [1], p.175.

The meaning of $[P, Q]$ for two conics of the contragredient variables. "A variable line which meets the conics in harmonically conjugate pairs of points, envelops another conic. This is called the harmonic envelope of the two conics."

The meaning of $[P, Q]$ for two conics from contragredient variables. "A variable point, from which the pairs of tangents to two given conics are harmonically conjugate to one another, describes another conic. This conic is called the harmonic locus of two conics."

Of course, $\widehat{A}$ is the envelope of $A$ (that is the set of tangents to $A$ ).

(6.2) Example. If a conic $A$ is written as a sum of three squares,

$$
A=a^{2}+b^{2}+c^{2}
$$

then the reciprocal conic of $A$ is

$$
\widehat{A}=[b c]^{2}+[a c]^{2}+[b c]^{2}
$$

and

$$
\operatorname{disc}(A)=<a . b \cdot c>^{2}
$$

(6.3) Example. If a conic $A$ is written as a sum of four squares,

$$
A=a^{2}+b^{2}+c^{2}+d^{2}
$$

then its discriminant also has a natural representation as a sum of four squares,

$$
\operatorname{disc}(A)=<a . b . c>^{2}+<a . b . d>^{2}+<a . c . d>^{2}+<b . c . d>^{2},
$$

the reciprocal conic of $A$ has a natural representation as a sum of six squares,

$$
\widehat{A}=[a, b]^{2}+[a, c]^{2}+[a, d]^{2}+[b, c]^{2}+[b, d]^{2}+[c, d]^{2},
$$

but there are possibilities (four in general) for writing $\widehat{A}$ as a sum of four squares (for the case of linear independence of every three from the four linear forms). One of 
them is

$$
\begin{aligned}
&<a . b . c>^{2} \widehat{A}=\operatorname{Disc}(A)\left([a . b]^{2}+[a, c]^{2}+[b, c]^{2}\right) \\
&-(<a . b . d>[a, b]+<a . c . d>[a, c]+<b . c . d>[b, c])^{2} .
\end{aligned}
$$

Three other representations can be derived by cyclic permutations of the linear forms $a, b, c, d$.

(6.4) Theorem. Any power of a proper quadratic form is an idempotent, more precisely, in the bialgebra $S^{2 n}(W)$, the following identities hold.

$$
\widehat{A^{n}}=c(n)(\widehat{A})^{n}, \quad \widehat{A^{n}}=c(n)^{3}(\operatorname{disc}(A))^{n} A^{n},<A^{n}, \widehat{A}^{n}>=(2 n+1) \operatorname{disc}(A)^{n},
$$

where $c(n)$ is a nonzero constant depending on $n$ (the constant is expressible in terms of binomial coefficients, but we will not need an exact description).

For example

$$
c(1)=1, c(2)=4 / 3, c(3)=8 / 5, c(4)=64 / 35 .
$$

In further parts of the text we will need obvious cases $n=1, n=2$ only, therefore proof is omitted.

(6.5) Theorem. For four ternary quadratic forms $A, B, C, D$ of the cogredient variables, we have the following identity

$$
\begin{aligned}
& {[[A, B],[C, D]]+[[A, C],[B, D]]+[[A, D],[B, C]] } \\
= & \langle B, C, D>A+<A, C, D>B+<A, B, D>C+<A, B, C>D .
\end{aligned}
$$

Proof. Because of the linearity with respect to every form, we may suppose that the forms are pure squares, and in the latter case, one can use (5.5).

(6.6) Remark. The Clebsch difference, the Clebsch covariant, the catalecticant. The identity of Theorem (6.5) for conics lead us to a consideration of the difference

$$
\begin{aligned}
\mathrm{Cl}(a, b, c, d)= & {[[a, b],[c, d]]+[[a, c],[b, d]]+[[a, d],[b, c]] } \\
& -(a<b, c, d>+b\langle a, c, d>+c<a, b, d\rangle+d<a, b, c\rangle)
\end{aligned}
$$

for four elements $a, b, c, d$ of arbitrary symmetric algebra $W$. One can call it the Clebsch difference. The Clebsch difference is the polarization of a function $S: W \rightarrow$ $W$, that is

$$
S(a)=\frac{1}{4 !} \mathrm{Cl}(a, a, a, a)=\frac{1}{2}(\widehat{\widehat{a}}-I(a) a) .
$$

In the algebra of ternary quadratic forms, this difference is identically zero, but if $a$ is a ternary quartic, then its Clebsch covariant coincides with the ordinary Clebsch covariant $S$ (considered in [5]).

One can introduce a notion of catalecticant $J(a)$ of an element $a$ of a symmetric bialgebra:

$$
J(a)=\frac{1}{36}<S(a) \cdot a \cdot a>
$$

Also, the catalecticant is expressible with the help of the cubic invariant $I$ (from Notation (2.5)) and the reciprocity operation (1.2) in the bialgebra:

$$
J(a)=\frac{1}{12}\left(I(\widehat{a})-I^{2}(a)\right) .
$$


For example, identity (5.6) together with Example (8.6) have as a corollary Clebsch-Lüroth's well-known formula for the catalecticant of a sum of four fourth powers of ternary linear forms.

List of identities for ternary quadratic forms. The identities below are either easy for verifying or follow from the identity of Theorem (6.5).

For quadratic forms $A, B, C$, we have

$$
\begin{aligned}
& \widehat{A+B}=\widehat{A}+[A, B]+\widehat{B}, \\
& <A,[A, B]>=2<\widehat{A}, B>, \\
& {[A,[\widehat{A}, \widehat{B}]]=\operatorname{disc}(A) \widehat{B}+<A, \widehat{B}>\widehat{A},}
\end{aligned}
$$

(6.11) $\operatorname{disc}([A, B])=<A, \widehat{B}><B, \widehat{A}>-\operatorname{disc}(A) \operatorname{disc}(B)$,

(6.12) $\operatorname{disc}([\widehat{A}, \widehat{B}])=\operatorname{disc}(A) \operatorname{disc}(B)(<A, \widehat{B}><B, \widehat{A}>-\operatorname{disc}(A) \operatorname{disc}(B))$.

$$
[A, \widehat{[A, B]}]=<A, \widehat{B}>\widehat{A}+<B, \widehat{A}>[A, B]-\operatorname{disc}(A) \widehat{B}
$$

$$
<[A, B],[\widehat{A}, \widehat{B}]>=<A, \widehat{B}><B, \widehat{A}>+3 \operatorname{disc}(A) \operatorname{disc}(B),
$$

$<[A, B],[\widehat{C}, \widehat{B}]>+<[A, C],[B, C], \widehat{B}>$

$$
=<A, \widehat{B}><B, \widehat{C}>+<C, \widehat{B}><A, B, C>+3<A, \widehat{C}>\operatorname{disc}(B) \text {, }
$$

(6.16) $[\widehat{A}, \widehat{B}]+\widehat{[A, B]}=<A, \widehat{B}>A+<B, \widehat{A}>B$.

(6.17) $\widehat{[\widehat{A}, \widehat{B}]}=-\operatorname{disc}(A) \operatorname{disc}(B)[A, B]$

$$
+\operatorname{disc}(A)<A, \widehat{B}>\widehat{B}+\operatorname{disc}(B)<B, \widehat{A}>\widehat{A} .
$$

\section{Apolarity.}

(7.1) Definition. Self-polar triangle. Let $a, b, c$ be three linearly independent ternary linear forms (a base of the cogredient part $W$ of the basic threedimensional bialgebra $(W,[]),. \quad p=[b, c], \quad q=[a, c], \quad r=[a, b]$. For a proper conic $A$, these three linear forms $a, b, c$ constitute a self-polar triangle, if one of the following equivalent conditions is fulfilled.

(i) $<p q, A>=0, \quad<p r, A>=0, \quad\langle q r, A>=0$,

(ii) $\langle a b, \widehat{A}\rangle=0, \quad\langle b c, \widehat{A}\rangle=0, \quad<a c, \widehat{A}\rangle=0$.

(7.2) Remark. Geometrically speaking (according to Salmon's "Conic Sections" [24]): Three points are said to form a self-polar triangle, if polar of each point (with respect to the conic) is the line joining the other two.

Further, if two conics have four distinct points of intersection, then they have a unique common self-polar triangle (see Baker's "Plane Geometry" [1]). Algebraic treatment of this fact is connected with the following construction of a cubic covariant.

(7.3) Notation. Let $J(A, B, C)$ denote the Jacobian determinant of three ternary quadratic forms $A, B, C$.

(7.4) Remark. This Jacobian is a cubic form. If $A, B$ are two conics with four distinct points of intersection, then $J(A, B,[\widehat{A}, \widehat{B}])$ is a product of three linear forms, these linear forms constitute a common self-polar triangle for $A, B$, and this triangle is unique for $A, B$. 
(7.5) Definition. Apolarity. Two elements $a, b \in W$ of an arbitrary symmetric bialgebra $(W,[]$,$) are mutually apolar, if \langle a, \widehat{b}\rangle=0, \quad<b, \widehat{a}\rangle=0$. Further, $a, b$ are said to be nondegenerate mutually apolar elements, if they are mutually apolar and

$$
a \neq 0, b \neq 0, \widehat{a} \neq 0, \widehat{b} \neq 0, \widehat{\widehat{a}} \neq 0, \widehat{\widehat{b}} \neq 0 .
$$

(7.6) Notation. If $a, b$ are mutually apolar, then sometimes we write $a \perp b$.

The notion of mutually apolar conics is well-known, see Semple and Kneebone [26], Welchmann [30].

(7.7) Theorem.

(1) For nonsingular conics $A$ and $B, A \perp B$ if and only if $\widehat{A} \perp \widehat{B}$.

(2) If $A \perp B$, then

$<[A, B], A>=0,<[\widehat{A}, \widehat{B}], \widehat{A}>=0,<[\widehat{A},[A, B]], \widehat{A}>=0,<\widehat{[A, B]}, \widehat{A}>=0$.

(3) Any pair of mutually apolar nonsingular conics have four different points of intersections, such a pair is non-degenerate.

Proof. (1) is a consequence of idempotency of proper conics.

(2) follows from identities (6.8), (6.10), (6.16).

(3) is proved in the mentioned books [26], [30].

(7.8) Theorem. If $x_{0}, x_{1}, x_{2}$ are three linear forms such that $x_{0}, x_{1}, x_{2}$ is a common self-polar triangle for two mutually apolar nondegenerate quadratic forms, then one of the forms is proportional to

$$
x_{0}^{2}+\omega x_{1}^{2}+\omega^{2} x_{2}^{2}
$$

other is proportional to

$$
x_{0}^{2}+\omega^{2} x_{1}^{2}+\omega x_{2}^{2},
$$

where $\omega$ is a primitive cubic root of unity.

Proof. See Gordan, [16], IV, §2, page 475, also see [26] and [30].

(7.9) Definition. Canonical coordinates. The three linear forms $x_{0}, x_{1}, x_{2}$ from the last theorem will be called canonical coordinates for the nondegenerate pair of mutually apolar quadratic forms.

(7.10) Theorem. Any three mutually apolar nondegenerate conics can be reduced to the following three

$$
\begin{gathered}
a\left(x_{0}^{2}+\omega x_{1}^{2}+\omega^{2} x_{2}^{2}\right), \\
b\left(x_{0}^{2}+\omega^{2} x_{1}^{2}+\omega x_{2}^{2}\right), \\
l\left(x_{0}^{2}+x_{1}^{2}+x_{2}^{2}\right)+2 k\left(x_{1} x_{2}+x_{2} x_{0}+x_{0} x_{1}\right),
\end{gathered}
$$

where $a, b, k, l$ are constants, $a \neq 0, b \neq 0, l \neq k, l \neq-2 k$.

Proof. Also, it was proved by P.Gordan in [16], IV, $\S 5, \S 6$, page 476.

(7.11) Theorem of Gerbaldi. There exists a set of six mutually apolar linearly independent nondegenerate ternary quadratic forms.

Thus, the bialgebra $S^{2}(W)$ (where $\operatorname{dim} W=3$ ) is semisimple (note that all proper quadratic forms are idempotents according to (6.4) with $n=1$ ).

Proof. The principal part of the proof consists of a list of six conics. We will write down two lists, because both of them will be used in further parts of the text. 
The first list is composed by Francesco Gerbaldi [11-14], the second list is composed by Paul Gordan [16]. Verifying of mutual apolarity is an easy calculation for both the lists.

First of all, we fix a list of constants, which will be used here and further. Let

$$
\omega=\exp \left(\frac{2 \pi i}{3}\right)=\frac{-1+i \sqrt{3}}{2}, \quad r=\frac{-\sqrt{3}+i \sqrt{5}}{4}, \quad c=\frac{1+i \sqrt{15}}{4} .
$$

The conjugate numbers are

$$
\bar{\omega}=\frac{-1-i \sqrt{3}}{2}=\omega^{2}, \quad \bar{r}=\frac{-\sqrt{3}-i \sqrt{5}}{4}, \quad \bar{c}=\frac{1-i \sqrt{15}}{4} .
$$

Note that

$$
c=1+r \sqrt{3}, \quad 2 c^{2}-c+2=0 .
$$

One of possible (but insufficient) explanations of arising the field $\mathbb{Q}(\sqrt{-15})$ in the theory of conics and quartics is that there are four representations of 4 ! with the help of the factorial polynomial $x(x+1)(x+2)(x+3)$ and two of the representations generate the field, more precisely, the equation

$$
x(x+1)(x+2)(x+3)=24
$$

has the following four roots

$$
x_{1}=1, \quad x_{2}=-4, \quad x_{3}=\frac{-3+i \sqrt{15}}{2}, \quad x_{4}=\frac{-3-i \sqrt{15}}{2} .
$$

\section{GORDAN'S LIST}

Consider six Gordan's ternary quadratic forms from [16].

$$
\begin{aligned}
& K_{1}=x_{0}^{2}+\omega x_{1}^{2}+\omega^{2} x_{2}^{2}, \\
& K_{2}=x_{0}^{2}+\omega^{2} x_{1}^{2}+\omega x_{2}^{2}, \\
& K_{3}=r^{2}\left(x_{0}^{2}+x_{1}^{2}+x_{2}^{2}\right)+r \sqrt{3}\left(x_{1} x_{2}+x_{2} x_{0}+x_{0} x_{1}\right), \\
& K_{4}=r^{2}\left(x_{0}^{2}+x_{1}^{2}+x_{2}^{2}\right)+r \sqrt{3}\left(x_{1} x_{2}-x_{2} x_{0}-x_{0} x_{1}\right), \\
& K_{5}=r^{2}\left(x_{0}^{2}+x_{1}^{2}+x_{2}^{2}\right)+r \sqrt{3}\left(-x_{1} x_{2}+x_{2} x_{0}-x_{0} x_{1}\right), \\
& K_{6}=r^{2}\left(x_{0}^{2}+x_{1}^{2}+x_{2}^{2}\right)+r \sqrt{3}\left(-x_{1} x_{2}-x_{2} x_{0}+x_{0} x_{1}\right) .
\end{aligned}
$$

These six form are linearly independent, moreover, expressions of the basic quadratic monomials in terms of the six forms presented below.

$$
\begin{aligned}
& x_{0}^{2}=\frac{4 \bar{r}^{2}}{3}\left(2 r^{2} K_{1}+2 r^{2} K_{2}+K_{3}+K_{4}+K_{5}+K_{6}\right), \\
& x_{1}^{2}=\frac{4 \bar{r}^{2}}{3}\left(2 r^{2} \omega^{2} K_{1}+2 r^{2} \omega K_{2}+K_{3}+K_{4}+K_{5}+K_{6}\right), \\
& x_{2}^{2}=\frac{4 \bar{r}^{2}}{3}\left(2 r^{2} \omega K_{1}+2 r^{2} \omega^{2} K_{2}+K_{3}+K_{4}+K_{5}+K_{6}\right), \\
& x_{1} x_{2}=\frac{\bar{r} \sqrt{3}}{6}\left(K_{3}+K_{4}-K_{5}-K_{6}\right), \\
& x_{0} x_{2}=\frac{\bar{r} \sqrt{3}}{6}\left(K_{3}-K_{4}+K_{5}-K_{6}\right),
\end{aligned}
$$




$$
x_{0} x_{1}=\frac{\bar{r} \sqrt{3}}{6}\left(K_{3}-K_{4}-K_{5}+K_{6}\right)
$$

The coefficients of the reciprocal forms are conjugate (and of course, the contragredient variables are inserted instead of cogredient ones).

$$
\begin{aligned}
& L_{1}=\widehat{K_{1}}=u_{0}^{2}+\omega^{2} u_{1}^{2}+\omega u_{2}^{2}, \\
& L_{2}=\widehat{K_{2}}=u_{0}^{2}+\omega u_{1}^{2}+\omega^{2} u_{2}^{2}, \\
& L_{3}=\widehat{K_{3}}=\bar{r}^{2}\left(u_{0}^{2}+u_{1}^{2}+u_{2}^{2}\right)+\bar{r} \sqrt{3}\left(u_{1} u_{2}+u_{2} u_{0}+u_{0} u_{1}\right), \\
& L_{4}=\widehat{K_{4}}=\bar{r}^{2}\left(u_{0}^{2}+u_{1}^{2}+u_{2}^{2}\right)+\bar{r} \sqrt{3}\left(u_{1} u_{2}-u_{2} u_{0}-u_{0} u_{1}\right), \\
& L_{5}=\widehat{K_{5}}=\bar{r}^{2}\left(u_{0}^{2}+u_{1}^{2}+u_{2}^{2}\right)+\bar{r} \sqrt{3}\left(-u_{1} u_{2}+u_{2} u_{0}-u_{0} u_{1}\right), \\
& L_{6}=\widehat{K_{6}}=\bar{r}^{2}\left(u_{0}^{2}+u_{1}^{2}+u_{2}^{2}\right)+\bar{r} \sqrt{3}\left(-u_{1} u_{2}-u_{2} u_{0}+u_{0} u_{1}\right) .
\end{aligned}
$$

Of course, the forms $L_{i}$ constitute a base for contragredient quadratic forms, expressions of quadratic monomials in terms of the elements of the basis are written below.

$$
\begin{aligned}
& u_{0}^{2}=\frac{4 r^{2}}{3}\left(2 \bar{r}^{2} L_{1}+2 \bar{r}^{2} L_{2}+L_{3}+L_{4}+L_{5}+L_{6}\right), \\
& u_{1}^{2}=\frac{4 r^{2}}{3}\left(2 \bar{r}^{2} \omega L_{1}+2 \bar{r}^{2} \omega^{2} L_{2}+L_{3}+L_{4}+L_{5}+L_{6}\right), \\
& u_{2}^{2}=\frac{4 r^{2}}{3}\left(2 \bar{r}^{2} \omega^{2} L_{1}+2 \bar{r}^{2} \omega L_{2}+L_{3}+L_{4}+L_{5}+L_{6}\right), \\
& u_{1} u_{2}=\frac{r \sqrt{3}}{6}\left(L_{3}+L_{4}-L_{5}-L_{6}\right), \\
& u_{0} u_{2}=\frac{r \sqrt{3}}{6}\left(L_{3}-L_{4}+L_{5}-L_{6}\right), \\
& u_{0} u_{1}=\frac{r \sqrt{3}}{6}\left(L_{3}-L_{4}-L_{5}+L_{6}\right) .
\end{aligned}
$$

All the discriminants of $K_{i}$ and $L_{i}$ are equal to 1 . It is not hard to check the relations of mutual apolarity.

\section{GERBALDI'S LIST}

Consider the following six ternary quadratic forms $f_{1}, \ldots, f_{6}$ from [12]

$$
\begin{aligned}
& f_{1}=x_{0}^{2}+2 c x_{1} x_{2} \\
& f_{2}=\omega\left(x_{1}^{2}+2 c x_{0} x_{2}\right) \\
& f_{3}=x_{2}^{2}+2 c x_{0} x_{1} \\
& f_{4}=-\frac{1}{3}(1+2 c) \omega\left[x_{0}^{2}+x_{1}^{2}+x_{2}^{2}-c\left(x_{1} x_{2}+x_{2} x_{0}+x_{0} x_{1}\right)\right] \\
& f_{5}=-\frac{1}{3}(1+2 c)\left[x_{0}^{2}+\omega^{2} x_{1}^{2}+\omega x_{2}^{2}-c\left(x_{1} x_{2}+\omega^{2} x_{2} x_{0}+\omega x_{0} x_{1}\right)\right] \\
& f_{6}=-\frac{1}{3}(1+2 c) \omega\left[x_{0}^{2}+\omega x_{1}^{2}+\omega^{2} x_{2}^{2}-c\left(x_{1} x_{2}+\omega x_{2} x_{0}+\omega^{2} x_{1} x_{0}\right)\right] .
\end{aligned}
$$

These six forms are linearly independent. Expressions of the basic quadratic monomials in terms of Gerbaldi's six forms and expressions for reciprocal of the forms are 
disposed below.

$$
\begin{aligned}
& x_{0}^{2}=\frac{1}{9}\left(3 f_{1}-2(1-c)\left(\omega^{2} f_{4}+f_{5}+\omega^{2} f_{6}\right)\right), \\
& x_{1}^{2}=\frac{\omega^{2}}{9}\left(3 f_{2}-2(1-c)\left(f_{4}+\omega^{2} f_{5}+\omega^{2} f_{6}\right)\right), \\
& x_{2}^{2}=\frac{1}{9}\left(3 f_{3}-2(1-c)\left(\omega^{2} f_{4}+\omega^{2} f_{5}+f_{6}\right),\right. \\
& x_{1} x_{2}=\frac{1}{9 c}\left(3 f_{1}+(1-c)\left(\omega^{2} f_{4}+f_{5}+\omega^{2} f_{6}\right)\right), \\
& x_{2} x_{0}=\frac{\omega^{2}}{9 c}\left(3 f_{2}+(1-c)\left(f_{4}+\omega^{2} f_{5}+\omega^{2} f_{6}\right)\right), \\
& x_{0} x_{1}=\frac{1}{9 c}\left(3 f_{3}+(1-c)\left(\omega^{2} f_{4}+\omega^{2} f_{5}+f_{6}\right)\right) . \\
& \widehat{f}_{1}=-c\left(c u_{0}^{2}+2 u_{1} u_{2}\right), \\
& \widehat{f}_{2}=-c \omega^{2}\left(c u_{1}^{2}+2 u_{0} u_{2}\right) \\
& \widehat{f}_{3}=-c\left(c u_{2}^{2}+2 u_{0} u_{1}\right), \\
& \widehat{f_{4}}=\frac{1}{3}(c+2) \omega^{2}\left[c\left(u_{0}^{2}+u_{1}^{2}+u_{2}^{2}\right)-\left(u_{1} u_{2}+u_{2} u_{0}+u_{0} u_{1}\right)\right] \\
& \widehat{f_{5}}=\frac{1}{3}(c+2)\left[c\left(u_{0}^{2}+\omega u_{1}^{2}+\omega^{2} u_{2}^{2}\right)-\left(u_{1} u_{2}+\omega u_{2} u_{0}+\omega^{2} u_{0} u_{1}\right)\right], \\
& \widehat{f_{6}}=\frac{1}{3}(c+2) \omega^{2}\left[c\left(u_{0}^{2}+\omega^{2} u_{1}^{2}+\omega u_{2}^{2}\right)-\left(u_{1} u_{2}+\omega^{2} u_{2} u_{0}+\omega u_{0} u_{1}\right)\right] .
\end{aligned}
$$

Now, it is not hard to check the relations of mutual apolarity.

(7.12) Theorem. Any set of six mutually apolar ternary quadratic forms with the discriminant 1 is projectively equivalent to Gordan's six or its conjugate six by an automorphism of the field $\mathbb{Q}(r)$.

Proof. It is proved at page 478 of Gordan's paper [16 ].

(7.13) Theorem. If $A, B, C$ are three distinct mutually apolar conics chosen from six mutually apolar conics, then

$$
<[A, B],[\widehat{A}, \widehat{C}]>=0, \quad<A \cdot A \cdot B>=0 .
$$

Proof. The last identity, which is an obvious corollary of

$$
<A \cdot A \cdot B>=<[A, A], B>=2<\widehat{A}, B>,
$$

is indicated by Gordan, see [16], page 493, where the equality $\left(K_{m} K_{m} K_{n}\right)^{2}=0(m \neq$ $n$ ) is written, this equality is equivalent to $\left\langle K_{m} \cdot K_{m} \cdot K_{n}\right\rangle=0$ (for $m \neq n$ ).

One could check the first identity, if we would have a list of all possible participating products. We present such a list for Gerbaldi's conics. Maybe according to J.E. Littlewood's "A Mathematician's Miscellany", p. 50, "One slip is practically certain in this style of writing, generally devil-inspired".

$$
\begin{aligned}
& 2 c\left[f_{1}, f_{2}\right]=\omega\left(\widehat{f}_{3}+\widehat{f}_{6}\right)+\omega^{2}\left(\widehat{f}_{4}+\widehat{f}_{5}\right), \\
& \left.2 c\left[f_{1}, f_{3}\right]=\omega\left(\widehat{f}_{2}+\widehat{f}_{4}\right)+\omega^{2} \widehat{f}_{5}+\widehat{f}_{6}\right), \\
& 2 c\left[f_{1}, f_{4}\right]=\omega\left(\widehat{f}_{3}+\widehat{f}_{5}\right)+\omega^{2}\left(\widehat{f}_{2}+\widehat{f}_{6}\right), \\
& 2 c\left[f_{1}, f_{5}\right]=\omega\left(\widehat{f}_{4}+\widehat{f}_{6}\right)+\omega^{2}\left(\widehat{f}_{2}+\widehat{f}_{3}\right),
\end{aligned}
$$




$$
\begin{aligned}
& 2 c\left[f_{1}, f_{6}\right]=\omega\left(\widehat{f_{2}}+\widehat{f}_{5}\right)+\omega^{2}\left(\widehat{f}_{3}+\widehat{f}_{4}\right), \\
& 2 c\left[f_{2}, f_{3}\right]=\omega\left(\widehat{f}_{1}+\widehat{f}_{5}\right)+\omega^{2}\left(\widehat{f}_{4}+\widehat{f}_{6}\right), \\
& 2 c\left[f_{2}, f_{4}\right]=\omega\left(\widehat{f}_{5}+\widehat{f}_{6}\right)+\omega^{2}\left(\widehat{f}_{1}+\widehat{f}_{3}\right), \\
& 2 c\left[f_{2}, f_{5}\right]=\omega\left(\widehat{f}_{3}+\hat{f}_{4}\right)+\omega^{2}\left(\hat{f}_{1}+\widehat{f}_{6}\right), \\
& 2 c\left[f_{2}, f_{6}\right]=\omega\left(\hat{f}_{1}+\widehat{f}_{4}\right)+\omega^{2}\left(\widehat{f}_{3}+\widehat{f}_{5}\right), \\
& 2 c\left[f_{3}, f_{4}\right]=\omega\left(\widehat{f}_{1}+\widehat{f}_{6}\right)+\omega^{2}\left(\widehat{f}_{2}+\widehat{f}_{5}\right), \\
& 2 c\left[f_{3}, f_{5}\right]=\omega\left(\hat{f}_{2}+\widehat{f}_{6}\right)+\omega^{2}\left(\widehat{f}_{1}+\widehat{f}_{4}\right), \\
& 2 c\left[f_{3}, f_{6}\right]=\omega\left(\hat{f}_{4}+\widehat{f}_{5}\right)+\omega^{2}\left(\hat{f}_{1}+\widehat{f}_{2}\right), \\
& 2 c\left[f_{4}, f_{5}\right]=\omega\left(\widehat{f}_{1}+\widehat{f}_{2}\right)+\omega^{2}\left(\widehat{f}_{3}+\widehat{f}_{6}\right), \\
& 2 c\left[f_{4}, f_{6}\right]=\omega\left(\widehat{f}_{2}+\widehat{f}_{3}\right)+\omega^{2}\left(\widehat{f}_{1}+\widehat{f}_{5}\right), \\
& 2 c\left[f_{5}, f_{6}\right]=\omega\left(\widehat{f}_{1}+\widehat{f}_{3}\right)+\omega^{2}\left(\widehat{f}_{2}+\widehat{f}_{4}\right) . \\
& -2\left[\widehat{f}_{1}, \widehat{f}_{2}\right]=c^{3}\left(\omega^{2}\left(f_{3}+f_{6}\right)+\omega\left(f_{4}+f_{5}\right)\right), \\
& -2\left[\widehat{f}_{1}, \widehat{f}_{3}\right]=c^{3}\left(\omega^{2}\left(f_{2}+f_{4}\right)+\omega\left(f_{5}+f_{6}\right)\right) \text {, } \\
& -2\left[\widehat{f}_{1}, \hat{f}_{4}\right]=c^{3}\left(\omega^{2}\left(f_{3}+f_{5}\right)+\omega\left(f_{2}+f_{6}\right)\right) \text {, } \\
& -2\left[\widehat{f}_{1}, \widehat{f}_{5}\right]=c^{3}\left(\omega^{2}\left(f_{4}+f_{6}\right)+\omega\left(f_{2}+f_{3}\right)\right) \text {, } \\
& -2\left[\widehat{f}_{1}, \widehat{f}_{6}\right]=c^{3}\left(\omega^{2}\left(f_{2}+f_{5}\right)+\omega\left(f_{3}+f_{4}\right)\right) \text {, } \\
& -2\left[\widehat{f}_{2}, \widehat{f}_{3}\right]=c^{3}\left(\omega^{2}\left(f_{1}+f_{5}\right)+\omega\left(f_{4}+f_{6}\right)\right) \text {, } \\
& -2\left[\widehat{f}_{2}, \widehat{f}_{4}\right]=c^{3}\left(\omega^{2}\left(f_{5}+f_{6}\right)+\omega\left(f_{1}+f_{3}\right)\right) \text {, } \\
& -2\left[\widehat{f}_{2}, \widehat{f}_{5}\right]=c^{3}\left(\omega^{2}\left(f_{3}+f_{4}\right)+\omega\left(f_{1}+f_{6}\right)\right) \text {, } \\
& -2\left[\widehat{f}_{2}, \widehat{f}_{6}\right]=c^{3}\left(\omega^{2}\left(f_{1}+f_{4}\right)+\omega\left(f_{3}+f_{5}\right)\right) \text {, } \\
& -2\left[\widehat{f}_{3}, \widehat{f}_{4}\right]=c^{3}\left(\omega^{2}\left(f_{1}+f_{6}\right)+\omega\left(f_{2}+f_{5}\right)\right) \text {, } \\
& -2\left[\widehat{f}_{3}, \widehat{f}_{5}\right]=c^{3}\left(\omega^{2}\left(f_{2}+f_{6}\right)+\omega\left(f_{1}+f_{4}\right)\right) \text {, } \\
& -2\left[\widehat{f}_{3}, \widehat{f}_{6}\right]=c^{3}\left(\omega^{2}\left(f_{4}+f_{5}\right)+\omega\left(f_{1}+f_{2}\right)\right) \text {, } \\
& -2\left[\widehat{f}_{4}, \widehat{f}_{5}\right]=c^{3}\left(\omega^{2}\left(f_{1}+f_{2}\right)+\omega\left(f_{3}+f_{6}\right)\right) \text {, } \\
& -2\left[\widehat{f}_{4}, \widehat{f}_{6}\right]=c^{3}\left(\omega^{2}\left(f_{2}+f_{3}\right)+\omega\left(f_{1}+f_{5}\right)\right) \text {, } \\
& -2\left[\widehat{f}_{5}, \widehat{f}_{6}\right]=c^{3}\left(\omega^{2}\left(f_{1}+f_{3}\right)+\omega\left(f_{2}+f_{4}\right)\right) \text {. }
\end{aligned}
$$

Note that for Gerbaldi's conics $f_{k}$ and $\widehat{f}_{k}$, we have

$$
\operatorname{disc}\left(f_{k}\right)=-c^{2}, \operatorname{disc}\left(\widehat{f}_{i}\right)=-c^{4}, \quad<f_{k}, \widehat{f}_{k}>=-3 c^{2},
$$

and, because of their mutual apolarity, $<f_{l}, \widehat{f}_{k}>=0$ (if $k \neq l$ ).

So, using the above identities, it is not hard to check that $<\left[f_{k}, f_{l}\right],\left[\widehat{f_{k}}, \widehat{f_{m}}\right]>=0$ for distinct indices $k, l, m$.

(7.14) Theorem. Let $A, B, C, D$ be four distinct conics from Gerbaldi's six, $c$ be the constant from their equations. There are a cubic root of unity $\epsilon$ and a square 
root of unity $\delta$ (the cases $\epsilon=1, \delta=1$ are not excluded) such that

$$
\begin{aligned}
<[A, B],[\widehat{C}, \widehat{D}]> & =\frac{3}{2} \epsilon^{2} c^{4}, \quad<[\widehat{A}, \widehat{B}],[C, D]>=\frac{3}{2} \epsilon c^{4}, \\
<A . C . D> & =\frac{3}{2} \epsilon \delta c, \quad<B . C . D>=\frac{3}{2} \epsilon \delta c, \\
<\widehat{A} . \widehat{B} . \widehat{C}> & =\frac{3}{2} \epsilon c^{5}, \quad<\widehat{A} \cdot \widehat{B} . \widehat{D}>=\frac{3}{2} \epsilon c^{5} .
\end{aligned}
$$

Proof. It will be an immediate verifying after some preliminary work over Gerbaldi's conics. First, we indicate list of equalities for these quadratic forms.

$$
\begin{aligned}
& <f_{1} \cdot f_{2} \cdot f_{3}>=<f_{1} \cdot f_{2} \cdot f_{6}>=<f_{1} \cdot f_{3} \cdot f_{4}>=<f_{1} \cdot f_{4} \cdot f_{5}>=<f_{1} \cdot f_{5} \cdot f_{6}> \\
& =<f_{2} \cdot f_{3} \cdot f_{5}>=<f_{2} \cdot f_{4} \cdot f_{5}>=<f_{2} \cdot f_{4} \cdot f_{6}>=<f_{3} \cdot f_{4} \cdot f_{6}>=<f_{3} \cdot f_{5} \cdot f_{6}> \\
& =-\frac{3}{2} \omega c, \\
& <f_{1} \cdot f_{2} \cdot f_{4}>=<f_{1} \cdot f_{2} \cdot f_{5}>=<f_{1} \cdot f_{3} \cdot f_{5}>=<f_{1} \cdot f_{3} \cdot f_{6}>=<f_{1} \cdot f_{4} \cdot f_{6}> \\
& =<f_{2} \cdot f_{3} \cdot f_{4}>=<f_{2} \cdot f_{3} \cdot f_{6}>=<f_{2} \cdot f_{5} \cdot f_{6}>=<f_{3} \cdot f_{4} \cdot f_{5}>=<f_{4} \cdot f_{5} \cdot f_{6}> \\
& =-\frac{3}{2} \omega^{2} c \text {, } \\
& <\widehat{f_{1}} \cdot \widehat{f_{2}} \cdot \widehat{f}_{4}>=<\widehat{f}_{1} \cdot \widehat{f}_{2} \cdot \widehat{f}_{5}>=<\widehat{f}_{1} \cdot \widehat{f}_{3} \cdot \widehat{f}_{5}>=<\widehat{f}_{1} \cdot \widehat{f}_{3} \cdot \widehat{f}_{6}>=<\widehat{f}_{1} \cdot \widehat{f}_{4} \cdot \widehat{f}_{6}> \\
& =<\widehat{f}_{2} \cdot \widehat{f}_{3} \cdot \widehat{f}_{4}>=<\widehat{f}_{2} \cdot \widehat{f}_{3} \cdot \widehat{f}_{6}>=<\widehat{f}_{2} \cdot \widehat{f}_{5} \cdot \widehat{f}_{6}>=<\widehat{f}_{3} \cdot \widehat{f}_{4} \cdot \widehat{f}_{5}>=<\widehat{f}_{4} \cdot \widehat{f}_{5} \cdot \widehat{f}_{6}> \\
& =\frac{3}{2} \omega c^{4} \\
& <\widehat{f_{1}} \cdot \widehat{f_{2}} \cdot \widehat{f_{3}}>=<\widehat{f}_{1} \cdot \widehat{f}_{2} \cdot \widehat{f}_{6}>=<\widehat{f_{1}} \cdot \widehat{f_{3}} \cdot \widehat{f}_{4}>=<\widehat{f_{1}} \cdot \widehat{f}_{4} \cdot \widehat{f}_{5}>=<\widehat{f}_{1} \cdot \widehat{f}_{5} \cdot \widehat{f}_{6}> \\
& =<\widehat{f}_{2} \cdot \widehat{f}_{3} \cdot \widehat{f}_{5}>=<\widehat{f}_{2} \cdot \widehat{f}_{4} \cdot \widehat{f}_{5}>=<\widehat{f}_{2} \cdot \widehat{f}_{4} \cdot \widehat{f}_{6}>=<\widehat{f}_{3} \cdot \widehat{f}_{4} \cdot \widehat{f}_{6}>=<\widehat{f}_{3} \cdot \widehat{f}_{5} \cdot \widehat{f}_{6}> \\
& =\frac{3}{2} \omega^{2} c^{4}
\end{aligned}
$$

For a description of numbers $<\left[f_{i}, f_{j}\right],\left[\widehat{f}_{k}, \widehat{f}_{l}\right]>$, one can write down the following auxiliary list of ordered triples of unordered pairs.

$$
\begin{array}{llll}
(\{1,2\},\{3,6\},\{4,5\}), & (\{3,6\},\{4,5\},\{1,2\}), & (\{4,5\},\{1,2\},\{3,6\}), \\
(\{1,3\},\{2,4\},\{5,6\}), & (\{2,4\},\{5,6\},\{1,3\}), & (\{5,6\},\{1,3\},\{2,4\}), \\
(\{1,4\},\{3,5\},\{2,6\}), & (\{3,5\},\{2,6\},\{1,4\}), & (\{2,6\},\{1,4\},\{3,5\}), \\
(\{1,5\},\{4,6\},\{2,3\}), & (\{4,6\},\{2,3\},\{1,5\}), & (\{2,3\},\{1,5\},\{4,6\}), \\
(\{1,6\},\{2,5\},\{3,4\}), & (\{2,5\},\{3,4\},\{1,6\}), & (\{3,4\},\{1,6\},\{2,5\}) .
\end{array}
$$

If $\{i, j\},\{k, l\}$ are ordered (in this sequence) neighbors in a triple of the list or in a triple derived from the list triple by a cyclic permutation of pairs, then

$$
<\left[f_{i}, f_{j}\right],\left[\widehat{f}_{k}, \widehat{f}_{l}\right]>=\frac{3}{2} \omega c^{4}, \quad<\left[f_{k}, f_{l}\right],\left[\widehat{f}_{i}, \widehat{f}_{j}\right]>=\frac{3}{2} \omega^{2} c^{4}
$$

else

$$
<\left[f_{i}, f_{j}\right],\left[\widehat{f}_{k}, \widehat{f}_{l}\right]>=\frac{3}{2} c^{4}
$$


For example,

$$
\begin{gathered}
<\left[f_{1}, f_{2}\right],\left[\widehat{f}_{4}, \widehat{f}_{5}\right]>=\frac{3}{2} \omega^{2} c^{4}, \quad<\left[f_{1}, f_{2}\right],\left[\widehat{f}_{3}, \widehat{f}_{6}\right]>=\frac{3}{2} \omega c^{4} \\
<\left[f_{1}, f_{3}\right],\left[\widehat{f}_{5}, \widehat{f}_{6}\right]>=\frac{3}{2} \omega^{2} c^{4}, \quad<\left[f_{1}, f_{3}\right],\left[\widehat{f}_{2}, \widehat{f}_{4}\right]>=\frac{3}{2} \omega c^{4} \\
<\left[f_{1}, f_{2}\right],\left[\widehat{f}_{3}, \widehat{f}_{4}\right]>=\frac{3}{2} c^{4}, \quad<\left[f_{1}, f_{2}\right],\left[\widehat{f}_{4}, \widehat{f}_{6}\right]>=\frac{3}{2} c^{4} \\
<\left[f_{1}, f_{2}\right],\left[\widehat{f}_{3}, \widehat{f}_{5}\right]>=\frac{3}{2} c^{4}, \quad<\left[f_{1}, f_{3}\right],\left[\widehat{f}_{2}, \widehat{f}_{5}\right]=\frac{3}{2} c^{4} \\
<\left[f_{1}, f_{3}\right],\left[\widehat{f}_{2}, \widehat{f}_{6}\right]>=\frac{3}{2} c^{4}, \quad<\left[f_{1}, f_{3}\right],\left[\widehat{f}_{4}, \widehat{f}_{5}\right]>=\frac{3}{2} c^{4}
\end{gathered}
$$

More complete information about these numbers (multiplied by $2 c^{-4}$ ) is presented by the $15 \times 15$ matrix from the proof of Theorem (7.14) below.

Now, verifying of the first two identities (and their concordance with four other ones) of the theorem is easy.

(7.14) Theorem. Let $A_{1}, A_{2}, A_{3}, A_{4}, A_{5}, A_{6}$ be six mutually apolar ternary quadratic forms.

(i) Fifteen decomposable quartics $A_{i} A_{j}, \quad(i \neq j, \quad i, j \in\{1,2,3,4,5,6\})$, are linearly independent, therefore they constitute a base for the linear space $S^{4}(W)$ of ternary quartics.

(ii) Fifteen quartics $\left[A_{i}, A_{j}\right]^{2}, \quad(i \neq j)$, are linearly independent, therefore they constitute a base for the linear space $S^{4}\left(W^{*}\right)$ of (contragredient) ternary quartics.

(iii) Fifteen quartics $\left[\widehat{A_{i}}, \widehat{A_{j}}\right]^{2}(i \neq j)$ are linearly independent, therefore they constitute a base for the linear space $S^{4}(W)$ of ternary quartics.

Proof. We will consider Gerbaldi's conics $f_{1}, \ldots, f_{6}$.

Proof of (i). According to the formulas expressing quadratic monomials in the terms of $f_{i}$, every quartic monomial is expressible in terms of $f_{i}^{2}, f_{k} f_{l}$. Therefore, it is enough to express the squares $f_{i}^{2}$ in terms of products of distinct forms. The necessary formulas are the following.

$$
\begin{gathered}
-2 \bar{c} f_{1}^{2}= \\
\omega\left(f_{2} f_{4}+f_{4} f_{6}+f_{6} f_{3}+f_{3} f_{5}+f_{5} f_{2}\right)+ \\
\omega^{2}\left(f_{2} f_{3}+f_{3} f_{4}+f_{4} f_{5}+f_{5} f_{6}+f_{6} f_{2}\right) \\
-2 \bar{c} f_{2}^{2}= \\
\omega\left(f_{1} f_{4}+f_{4} f_{3}+f_{3} f_{6}+f_{6} f_{5}+f_{5} f_{1}\right)+ \\
\omega^{2}\left(f_{1} f_{3}+f_{3} f_{5}+f_{5} f_{4}+f_{4} f_{6}+f_{6} f_{1}\right), \\
-2 \bar{c} f_{3}^{2}= \\
\omega\left(f_{1} f_{5}+f_{5} f_{4}+f_{4} f_{2}+f_{2} f_{6}+f_{6} f_{1}\right)+ \\
\omega^{2}\left(f_{1} f_{2}+f_{2} f_{5}+f_{5} f_{6}+f_{6} f_{4}+f_{4} f_{1}\right) \\
-2 \bar{c} f_{4}^{2}= \\
\omega\left(f_{1} f_{2}+f_{2} f_{3}+f_{3} f_{5}+f_{5} f_{6}+f_{6} f_{1}\right)+
\end{gathered}
$$




$$
\begin{aligned}
& \omega^{2}\left(f_{1} f_{3}+f_{3} f_{6}+f_{6} f_{2}+f_{2} f_{5}+f_{5} f_{1}\right), \\
& -2 \bar{c} f_{5}^{2}= \\
& \omega\left(f_{1} f_{2}+f_{2} f_{6}+f_{6} f_{4}+f_{4} f_{3}+f_{3} f_{1}\right)+ \\
& \omega^{2}\left(f_{1} f_{4}+f_{4} f_{2}+f_{2} f_{3}+f_{3} f_{6}+f_{6} f_{1}\right), \\
& -2 \bar{c} f_{6}^{2}= \\
& \omega\left(f_{1} f_{3}+f_{3} f_{2}+f_{2} f_{5}+f_{5} f_{4}+f_{4} f_{1}\right)+ \\
& \omega^{2}\left(f_{1} f_{2}+f_{2} f_{4}+f_{4} f_{3}+f_{3} f_{5}+f_{5} f_{1}\right) \text {. }
\end{aligned}
$$

Proof of (ii). It is enough to prove that $15 \times 15$ matrix $A$ of mutual scalar products $<f_{i} f_{j},\left[\widehat{f}_{k}, \widehat{f}_{l}\right]>$, where the pairs $\{i, j\},\{k, l\}$ run the set

$$
\begin{gathered}
(1,2\},\{1,3\},\{1,4\},\{1,5\},\{1,6\},\{2,3\},\{2,4\},\{2,5\}, \\
\{2,6\},\{3,4\},\{3,5\},\{3,6\},\{4,5\},\{4,6\},\{5,6\},
\end{gathered}
$$

is invertible. This matrix, multiplied by $2 c^{-4}$ is the following.

$$
\left(\begin{array}{ccccccccccccccc}
4 & 0 & 0 & 0 & 0 & 0 & 0 & 0 & 0 & 3 & 3 & 3 \omega & 3 \bar{\omega} & 3 & 3 \\
0 & 4 & 0 & 0 & 0 & 0 & 3 \omega & 3 & 3 & 0 & 0 & 0 & 3 & 3 & 3 \bar{\omega} \\
0 & 0 & 4 & 0 & 0 & 3 & 0 & 3 & 3 \bar{\omega} & 0 & 3 \omega & 3 & 0 & 0 & 3 \\
0 & 0 & 0 & 4 & 0 & 3 \bar{\omega} & 3 & 0 & 3 & 3 & 0 & 3 & 0 & 3 \omega & 0 \\
0 & 0 & 0 & 0 & 4 & 3 & 3 & 3 \omega & 0 & 3 \bar{\omega} & 3 & 0 & 3 & 0 & 0 \\
0 & 0 & 3 & 3 \omega & 3 & 4 & 0 & 0 & 0 & 0 & 0 & 0 & 3 & 3 \bar{\omega} & 3 \\
0 & 3 \bar{\omega} & 0 & 3 & 3 & 0 & 4 & 0 & 0 & 0 & 3 & 3 & 0 & 0 & 3 \omega \\
0 & 3 & 3 & 0 & 3 \bar{\omega} & 0 & 0 & 4 & 0 & 3 \omega & 0 & 3 & 0 & 3 & 0 \\
0 & 3 & 3 \omega & 3 & 0 & 0 & 0 & 0 & 4 & 3 & 3 \bar{\omega} & 0 & 3 & 0 & 0 \\
3 & 0 & 0 & 3 & 3 \omega & 0 & 0 & 3 \bar{\omega} & 3 & 4 & 0 & 0 & 0 & 0 & 3 \\
3 & 0 & 3 \bar{\omega} & 0 & 3 & 0 & 3 & 0 & 3 \omega & 0 & 4 & 0 & 0 & 3 & 0 \\
3 \bar{\omega} & 0 & 3 & 3 & 0 & 0 & 3 & 3 & 0 & 0 & 0 & 4 & 3 \omega & 0 & 0 \\
3 \omega & 3 & 0 & 0 & 3 & 3 & 0 & 0 & 3 & 0 & 0 & 3 \bar{\omega} & 4 & 0 & 0 \\
3 & 3 & 0 & 3 \bar{\omega} & 0 & 3 \omega & 0 & 3 & 0 & 0 & 3 & 0 & 0 & 4 & 0 \\
3 & 3 \omega & 3 & 0 & 0 & 3 & 3 \bar{\omega} & 0 & 0 & 3 & 0 & 0 & 0 & 0 & 4
\end{array}\right)
$$

A calculation of its determinant gives the following result.

$$
\operatorname{det}(A)=-2471326208=-2^{9} \cdot 13^{6} .
$$

Thus, (ii) is proved.

Proof of (iii). It is an evident consequence of (ii).

(7.15) Theorem. Let $A, A^{\prime}$ be two conics belonging to a set of six mutually apolar conics, $k, l, m, n$ be four bitangents for the pair $A, A^{\prime}$. Then every of the eight points of contact belongs to some other (distinct from $A$ and $A^{\prime}$ ) conic of the set.

Proof. We will consider Gordan's conics $K_{i}$ from proof of Theorem (7.11). Because of double transitivity of the induced action of Valentiner's group on the set of conics, we may suppose that $A=K_{1}, A^{\prime}=K_{2}$. It is not hard to see, that up to a permutation of the bitangents (and up to proportionality),

$$
\begin{aligned}
& k=x_{0}-x_{1}-x_{2}, \quad l=-x_{0}+x_{1}-x_{2}, \\
& m=-x_{0}-x_{1}+x_{2}, \quad n=x_{0}+x_{1}+x_{2} .
\end{aligned}
$$


The eight points of contact are the following.

$$
\begin{aligned}
& u=\left(-1, \omega^{2}, \omega\right), \quad v=\left(1,-\omega^{2}, \omega\right), \quad w=\left(1, \omega^{2},-\omega\right), \quad z=\left(1, \omega^{2}, \omega\right), \\
& u^{\prime}=\left(-1, \omega, \omega^{2}\right), \quad v^{\prime}=\left(1,-\omega, \omega^{2}\right), \quad w^{\prime}=\left(1, \omega, \omega^{2}\right), \quad z^{\prime}=\left(1, \omega, \omega^{2}\right) .
\end{aligned}
$$

Note that

$$
\begin{aligned}
& \{u\}=A \cap k,\{v\}=A \cap l,\{w\}=A \cap m,\{z\}=A \cap n, \\
& \left\{u^{\prime}\right\}=A^{\prime} \cap k,\left\{v^{\prime}\right\}=A^{\prime} \cap l,\left\{w^{\prime}\right\}=A^{\prime} \cap m,\left\{z^{\prime}\right\}=A^{\prime} \cap n,
\end{aligned}
$$

The points are indicated in paper [23] of R. Rodríguez and V.González-Aguilera, although they described the points with another connection (see page 53 and Figure 5 of page 54 in [23], where one can see bitangents to some quartics of a pencil, the pencil contains a decomposable quartic $K_{1} K_{2}$ ).

Now, one can see that

$$
\begin{aligned}
& u \in K_{1} \cap K_{4}, \quad v \in K_{1} \cap K_{5}, \\
& w \in K_{1} \cap K_{6}, \quad z \in K_{1} \cap K_{3}, \\
& u^{\prime} \in K_{2} \cap K_{4}, \quad v^{\prime} \in K_{2} \cap K_{5}, \\
& w^{\prime} \in K_{2} \cap K_{6}, \quad z^{\prime} \in K_{2} \cap K_{3} .
\end{aligned}
$$

These relations finish the proof.

8. The bialgebra of ternary quartics.

(8.1) The bimultiplication formulas. Ternary quartic form is

$$
\begin{gathered}
F\left(x_{0}, x_{1}, x_{2}\right)=\sum a_{i j k l} x_{i} x_{j} x_{k} x_{l}= \\
a_{0000} x_{0}^{4}+a_{1111} x_{1}^{4}+a_{2222} x_{2}^{4}+6 a_{1122} x_{1}^{2} x_{2}^{2}+6 a_{0022} x_{0}^{2} x_{2}^{2}+6 a_{0011} x_{0}^{2} x_{1}^{2}+ \\
12 a_{0012} x_{0}^{2} x_{1} x_{2}+12 a_{0112} x_{0} x_{1}^{2} x_{2}+12 a_{0112} x_{0} x_{1} x_{2}^{2}+ \\
4 a_{0001} x_{0}^{3} x_{1}+4 a_{0002} x_{0}^{3} x_{2}+4 a_{1110} x_{0} x_{1}^{3}+4 a_{1112} x_{1}^{3} x_{2}+4 a_{2220} x_{0} x_{2}^{3}+4 a_{2221} x_{1} x_{2}^{3} .
\end{gathered}
$$

If $G$ is another quartic form with coefficients $b_{i j k l}$, and

$$
[F, G]=Q\left(u_{0}, u_{1}, u_{2}\right)=\sum q_{i j k l} u_{i} u_{j} u_{k} u_{l}
$$

then

$$
\begin{gathered}
q_{0000}=a_{1111} b_{2222}+a_{2222} b_{1111}+6 a_{1122} b_{1122}-4 a_{1112} b_{2221}-4 a_{2221} b_{1112} \\
q_{1111}=a_{0000} b_{2222}+a_{2222} b_{0000}+6 a_{0022} b_{0022}-4 a_{0002} b_{2220}-4 a_{2220} b_{0002} \\
q_{2222}=a_{0000} b_{1111}+a_{1111} b_{0000}+6 a_{0011} b_{0011}-4 a_{0001} b_{1110}-4 a_{1110} b_{0001} \\
q_{1122}=a_{0000} b_{1122}+a_{1122} b_{0000}+a_{0022} b_{0011}+a_{0011} b_{0022}+ \\
4 a_{0012} b_{0012}-2 a_{0001} b_{0122}-2 a_{0122} b_{0001}-2 a_{0002} b_{0112}-2 a_{0112} b_{0002} \\
q_{0022}=a_{1111} b_{0022}+a_{0022} b_{1111}+a_{0011} b_{1122}+a_{1122} b_{0011}+ \\
4 a_{0112} b_{0112}-2 a_{1112} b_{0012}-2 a_{0012} b_{1112}-2 a_{1110} b_{0122}-2 a_{0122} b_{1110} \\
q_{0011}=a_{2222} b_{0011}+a_{0011} b_{2222}+a_{1122} b_{0022}+a_{0022} b_{1122}+
\end{gathered}
$$




$$
\begin{aligned}
& 4 a_{0122} b_{0122}-2 a_{2220} b_{0112}-2 a_{0112} b_{2220}-2 a_{2221} b_{0012}-2 a_{0012} b_{2221}, \\
& q_{0001}=3 a_{0112} b_{2221}+3 a_{2221} b_{0112}-3 a_{0122} b_{1122}-3 a_{1122} b_{0122} \\
& -a_{2222} b_{1110}-a_{1110} b_{2222}+a_{1112} b_{2220}+a_{2220} b_{1112}, \\
& q_{0002}=3 a_{0122} b_{1112}+3 a_{1112} b_{0122}-3 a_{0112} b_{1122}-3 a_{1122} b_{0112} \\
& -a_{1111} b_{2220}-a_{2220} b_{1111}+a_{2221} b_{1110}+a_{1110} b_{2221}, \\
& q_{1110}=3 a_{0012} b_{2220}+3 a_{2220} b_{0012}-3 a_{0122} b_{0022}-3 a_{0022} b_{0122} \\
& -a_{2222} b_{0001}-a_{0001} b_{2222}+a_{0002} b_{2221}+a_{2221} b_{0002}, \\
& q_{1112}=3 a_{0122} b_{0002}+3 a_{0002} b_{0122}-3 a_{0012} b_{0022}-3 a_{0022} b_{0012} \\
& -a_{0000} b_{2221}-a_{2221} b_{0000}+a_{2220} b_{0001}+a_{0001} b_{2220}, \\
& q_{2220}=3 a_{0012} b_{1110}+3 a_{1110} b_{0012}-3 a_{0112} b_{0011}-3 a_{0011} b_{0112} \\
& -a_{1111} b_{0002}-a_{0002} b_{1111}+a_{0001} b_{1112}+a_{1112} b_{0001}, \\
& q_{2221}=3 a_{0112} b_{0001}+3 a_{0001} b_{0112}-3 a_{0012} b_{0011}-3 a_{0011} b_{0112} \\
& -a_{0000} b_{1112}-a_{1112} b_{0000}+a_{1110} b_{0002}+a_{0002} b_{1110}, \\
& q_{0012}=2 a_{1122} b_{0012}+2 a_{0012} b_{1122}-a_{0112} b_{0122}-a_{0122} b_{0112} \\
& -a_{0022} b_{1112}-a_{1112} b_{0022}-a_{0011} b_{2221}-a_{2221} b_{0011} \\
& +a_{1110} b_{2220}+a_{2220} b_{1110} \\
& q_{0112}=2 a_{0022} b_{0112}+2 a_{0112} b_{0022}-a_{0012} b_{0122}-a_{0122} b_{0012} \\
& -a_{1122} b_{0002}-a_{0002} b_{1122}-a_{0011} b_{2220}-a_{2220} b_{0011} \\
& +a_{0001} b_{2221}+a_{2221} b_{0001}, \\
& q_{0122}=2 a_{0011} b_{0122}+2 a_{0122} b_{0011}-a_{0112} b_{0012}-a_{0012} b_{0112} \\
& -a_{0022} b_{1110}-a_{1110} b_{0022}-a_{0022} b_{1112}-a_{1112} b_{0022} \\
& +a_{1112} b_{0002}+a_{0002} b_{1112} \text {. }
\end{aligned}
$$

These formulas are produced by bilinearization of formulas from No. 292 of "Treatise" [25] by Salmon. The trilinear potential of the bimultiplication on quartics is a polarization of the so called invariant of third degree (with respect to the coefficients). More precisely, if $F, G, H$ are three quartics, then the potential is

$$
<F . G . H>\quad(=<[F, G], H>=<[G, H], F>=<[H, F], G>),
$$


the corresponding invariant is

$$
I(F)=\frac{1}{3 !}<F . F . F>=\frac{1}{3}<\sigma(F), F>.
$$

Expression of the cubic invariant in terms of the coefficients of $F$ is given in No. 293 of [25], the invariant is as follows.

$$
\begin{gathered}
I(F)=a_{0000} a_{1111} a_{2222} \\
+4\left(a_{0001} a_{1112} a_{2220}+a_{0002} a_{2221} a_{1110}\right) \\
+3\left(a_{0000} a_{1122}^{2}+a_{1111} a_{0022}^{2}+a_{2222} a_{0011}^{2}\right) \\
-4\left(a_{0000} a_{1112} a_{2221}+a_{1111} a_{0002} a_{2220}+a_{2222} a_{0001} a_{1110}\right) \\
-12\left(a_{0001} a_{0122} a_{1122}+a_{0002} a_{0112} a_{1122}+a_{1110} a_{0122} a_{0022}\right) \\
-12\left(a_{1112} a_{0012} a_{0022}+a_{2220} a_{0112} a_{0011}+a_{2221} a_{0012} a_{0011}\right) \\
+12\left(a_{0012} a_{0111} a_{0222}+a_{0112} a_{1222} a_{1000}+a_{0122} a_{2000} a_{2111}\right) \\
+12\left(a_{0011} a_{0122}^{2}+a_{0022} a_{0112}^{2}+a_{1122} a_{0012}^{2}\right) \\
-12 a_{0012} a_{0112} a_{0122}+6 a_{0011} a_{1122} a_{2200} .
\end{gathered}
$$

It is clear that

$$
q_{k l m n}=\sum_{p q r s} \frac{\partial^{2} I(F)}{\partial a_{i j k l} \partial a_{p q r s}} b_{p q r s}=\sum_{p q r s} \frac{\partial^{2} I(G)}{\partial b_{i j k l} \partial b_{p q r s}} a_{p q r s} .
$$

Note that

$$
\begin{gathered}
I(F+G+H)=<F \cdot G \cdot H>+I(F)+I(G)+I(H)+ \\
<\sigma(F), G>+<F, \sigma(G)>+<\sigma(F), H>+ \\
<F, \sigma(H)>+<\sigma(G), H>+<G, \sigma(H)>. \\
I(F+G)=I(F)+<\sigma(F), G>+<F, \sigma(G)>+I(G) .
\end{gathered}
$$

(8.2) Example. If $F=a b c d$ is a product of four ternary linear forms, then

$$
\begin{aligned}
\sigma(a b c d) & =\frac{1}{24}\left([a, b]^{2}[c, d]^{2}+[a, c]^{2}[b, d]^{2}+[a, d]^{2}[b, c]^{2}\right), \\
I(F) & =\frac{1}{144}<\text { a.b.c }><\text { a.c.d }><\text { a.c. } d><\text { b.c.d }>.
\end{aligned}
$$

The last expression is the so called symbolic form of the cubic invariant $I$.

Moreover, $\sigma(a b c d)$ is a product of two quadratic forms. One can see it with the help of coordinates.

$$
\begin{aligned}
\sigma\left(x_{0} x_{1} x_{2}\left(x_{0}+x_{1}+x_{2}\right)\right) & =2\left(u_{1}^{2} u_{2}^{2}+u_{2}^{2} u_{0}^{2}+u_{0}^{2} u_{1}^{2}-u_{0}^{2} u_{1} u_{2}-u_{1}^{2} u_{2} u_{0}-u_{2}^{2} u_{0} u_{1}\right) \\
& =2\left(u_{1} u_{2}+\omega u_{2} u_{0}+\omega^{2} u_{0} u_{1}\right)\left(u_{1} u_{2}+\omega^{2} u_{2} u_{0}+\omega u_{0} u_{1}\right),
\end{aligned}
$$

where $\omega$ is a primitive cubic root of unity.

The calculation has the following geometrical meaning. Four lines $a, b, c, d$ define a pencil of contragredient conics touching these four lines. This pencil has three degenerate conics. Two conics (of the pencil) equianharmonically disposed in the pencil with respect to the three degenerate conics are components of the $\sigma$-image of 
the four lines. These two conics are mutually apolar (according to formulas from $(7.8))$.

We recall the following definition.

Definition. Four points $p, q, r, s$ of the projective line form an equianharmonic quadruple, if their anharmonic ratio $(p, q, r, s)$ is a root of the equation $x^{2}-x+1=0$.

If three points $p, q, r \in \mathbb{P}^{1}$ are given, then there are two points $s \in \mathbb{P}^{1}$ exactly such that the quadruple $(p, q, r, s)$ (and all its permutations) is equianharmonic.

(8.3) Example. If $A=A\left(x_{0}, x_{1}, x_{2}\right)$ is a ternary quadratic form, then

$$
I\left(A^{2}\right)=\frac{20}{9}(\operatorname{disc}(A))^{2} .
$$

(8.4) Example. If $M\left(x_{0}, x_{1}, x_{2}\right)$ is a ternary cubic form then

$$
I\left(\left(u_{0} x_{0}+u_{1} x_{1}+u_{2} x_{2}\right) M\right)=4 \mathrm{Ca}(M)
$$

where $\mathrm{Ca}(M)=\mathrm{Ca}(M)\left(u_{0}, u_{1}, u_{2}\right)$ is the Cayley contravariant form of $M$.

(8.5) Example. (cf. Example (6.2)) If $F=a^{4}+b^{4}+c^{4}$, then

$$
\sigma(F)=[b, c]^{4}+[a, c]^{4}+[a, b]^{4}, I(F)=<a . b . c>^{4}, \sigma(\sigma(F))=I(F) F .
$$

Therefore, if $a, b, c$ are linearly independent, then $F$ is an idempotent.

(8.6) Example. (cf. Example (6.3)) If $F=a^{4}+b^{4}+c^{4}+d^{4}$, then

$$
\begin{gathered}
\sigma(F)=[a, b]^{4}+[a, c]^{4}+[a, d]^{4}+[b, c]^{4}+[b, d]^{4}+[c, d]^{4}, \\
I(F)=<a . b . c>^{4}+<a . b . d>^{4}+<a . c . d>^{4}+<b . c . d>^{4}, \\
\sigma(\sigma(F))=I(F) F+12<\text { a.b.c }><\text { a.b.d }><\text { a.c.d }><\text { b.c.d }>\text { abcd. }
\end{gathered}
$$

The last identity is a consequence of (5.6).

(8.7) Example. For the Bernoulli lemniscate, the contravariant $\sigma$ is a double conic .

First, about a projectively reasonable definition of the lemniscate. This is a plane quartic rational curve with three distinct nodal points, where all the six nodal tangents are inflexional (that is they intersect the curve only in nodes). One can give an equivalent description of the curve with the help of parameterization. For the normalization $\pi: \mathbb{P}^{1} \rightarrow C$ of $C$, the set of three pairs of points having singular $\pi$-images is invariant with respect to the tetrahedral fractional linear group of symmetries, the action admits an extension to the action on the projective plane. Up to a projective equivalence, such a curve is unique. Name of the curve is lemniscate (see Kohn and Loria [20], No. 94). Both the descriptions are projective characterizations of the Bernoulli lemniscate

$$
\left(x_{1}^{2}+x_{2}^{2}\right)^{2}-2 a^{2} x_{0}^{2}\left(x_{1}^{2}-x_{2}^{2}\right)=0
$$

with parameterization

$$
x_{0}=4 a^{4}+t^{4}, x_{1}=4 a^{4} t+2 a^{2} t^{3}, x_{2}=4 a^{4} t-2 a^{2} t^{3} .
$$

It is not hard to see that

$$
\sigma\left(3\left(x_{1}^{2}+x_{2}^{2}\right)^{2}-6 x_{0}^{2}\left(x_{1}^{2}-x_{2}^{2}\right)\right)=3\left(2 u_{0}^{2}-u_{1}^{2}+u_{2}^{2}\right)^{2} .
$$


For consideration of all 24 projective symmetries, more convenient equation of a projectively equivalent curve is

$$
x_{1}^{2} x_{2}^{2}+x_{2}^{2} x_{0}^{2}+x_{0}^{2} x_{1}^{2}=0 .
$$

Rational parameterization of the latter curve is

$$
x_{0}=2 i t^{2}\left(t^{2}-1\right), x_{1}=2 t^{2}\left(t^{2}+1\right), x_{2}=t^{4}-1,
$$

the fractional linear tetrahedral group is described in Ford ([8], No. 55), or in F. Klein [20], p.137, formulas (4a), (4b), (4c).

The corresponding collineation group consists of transformations

$$
x_{0}^{\prime}= \pm x_{\alpha}, x_{1}^{\prime}= \pm x_{\beta}, x_{2}^{\prime}= \pm x_{\alpha} .
$$

The contravariant $\sigma$ is a double conic,

$$
\sigma\left(6\left(x_{1}^{2} x_{2}^{2}+x_{2}^{2} x_{0}^{2}+x_{0}^{2} x_{1}^{2}\right)\right)=3\left(u_{0}^{2}+u_{1}^{2}+u_{2}^{2}\right)^{2} .
$$

9. The bialgebra calculus of decomposable quartics. Here, "decomposable" means a product of two quadratic ternary forms.

First, the identities describing pairings of some decomposable quartics.

If $A(x), B(x), C(x), D(x)$ are quadratic forms of $(x)=\left(x_{0}, x_{1}, x_{2}\right), P(u), Q(u)$ are quadratic forms of contragredient variables, then

$$
\begin{aligned}
& <P^{2}, A^{2}>=<P, A>^{2}-\frac{4}{3}<\widehat{P}, \widehat{A}> \\
& <P Q, A^{2}>=<P, A><Q, A>-\frac{2}{3}<[P, Q], \widehat{A}> \\
& <P Q, A B>=-\frac{1}{3}<[P, Q],[A, B]> \\
& \frac{1}{2}(<P, A><Q, B>+<P, B><Q, A>) \\
& <\widehat{C} \widehat{D}, A B>=-\frac{1}{3}<[\widehat{C}, \widehat{D}],[A, B]> \\
& +\frac{1}{2}<\widehat{C}, A><\widehat{D}, B>+\frac{1}{2}<\widehat{C}, B><\widehat{D}, A> \\
& <\widehat{C}, A B>=<\widehat{C}, A><\widehat{C}, B>-\frac{2}{3}<A . B . C>\operatorname{disc}(C), \\
& <\widehat{B} \widehat{C}, A^{2}>=<\widehat{B}, A><\widehat{C}, A>-\frac{2}{3}<\widehat{A} . \widehat{B} . \widehat{C}> \\
& <A^{2},[B, C]^{2}>=<A . B . C>2+\frac{4}{3}<\widehat{A} . \widehat{B} . \widehat{C}> \\
& -\frac{4}{3}<\widehat{A}, C><\widehat{B}, C>-\frac{4}{3}<\widehat{A}, B><\widehat{C}, B>
\end{aligned}
$$

$$
\begin{aligned}
<\widehat{A}^{2},[\widehat{B}, \widehat{C}]^{2} & >=<\widehat{A} \cdot \widehat{B} \cdot \widehat{C}>^{2}+\frac{4}{3} \operatorname{disc}(A) \operatorname{disc}(B) \operatorname{disc}(C)<A . B . C>+ \\
-\frac{4}{3} \operatorname{disc}(A) \operatorname{disc}(B) & <\widehat{C}, A><\widehat{C}, B>-\frac{4}{3} \operatorname{disc}(A) \operatorname{disc}(C)<\widehat{B}, A><\widehat{B}, C>
\end{aligned}
$$

$$
<[\widehat{A}, \widehat{B}]^{2},[C, D]^{2}>=<[\widehat{A}, \widehat{B}],[C, D]>^{2}-\frac{4}{3} \operatorname{disc}(A) \operatorname{disc}(B)<[A, B],[\widehat{C}, \widehat{D}]>
$$


Second, we write some identities with the bimultiplication of the decomposable forms.

If $A(x), B(x), C(x), D(x)$ are ternary quadratic forms, then

$$
\begin{aligned}
& {\left[A^{2}, B^{2}\right]=[A, B]^{2}-\frac{4}{3} \widehat{A} \widehat{B}} \\
& {[A B, A C]=\frac{1}{6}[A, B][A, C]+[B, C] \widehat{A}} \\
& {\left[C D, B^{2}\right]=[C, B][D, B]-\frac{2}{3}[C, D] \widehat{B}} \\
& {[A B, C D]=\frac{1}{2}[A, C][B, D]+\frac{1}{2}[A, D][B, C]-\frac{1}{3}[A, B][C, D]} \\
& \sigma(A B)=\frac{1}{12}[A, B]^{2}+\widehat{A} \widehat{B}, \\
& \sigma\left(A^{2}+B^{2}\right)=\frac{4}{3}\left(\widehat{A}^{2}+\widehat{B}^{2}\right)-\frac{4}{3} \widehat{A} \widehat{B}+[A, B]^{2} .
\end{aligned}
$$

10. Salmon subalgebra, Wiman subalgebra. In this section, we suppose that $W$ is the three-dimensional skew bialgebra. Let $a, b, c \in W$ be three linearly independent linear forms (basis of $W$ ). We will consider the extension bialgebra $\left(S^{2 m}(W),[],\right)$ and some its subalgebras.

(10.1) Notation. Let $U_{2 m}(a, b, c)$ be the set of forms (from $S^{2 m}(W)$ )

$$
a_{0} a^{2 m}+a_{1} b^{2 m}+a_{2} c^{2 m}+b_{0}(b c)^{m}+b_{1}(c a)^{m}+b_{2}(a b)^{m}
$$

where $a_{i}, b_{i}$ are elements of $K$. Let $V_{2 m}(a, b, c) \subset S^{2 m}\left(W^{*}\right)$ be the set of forms $p_{0}[b, c]^{2 m}+p_{1}[c, a]^{2 m}+p_{2}[a, b]^{2 m}+q_{0}([c, a][a, b])^{m}+q_{1}([a, b][b, c])^{m}+q_{2}([b, c][c, a])^{m}$, where $p_{i}, q_{i}$ are constants from $K$.

(10.2) Theorem. $\left(U_{2 m}(a, b, c), V_{2 m}(a, b, c)\right)$ is a subalgebra of $\left(S^{2 m}(W),[],\right)$. Proof. It follows from formulas (5.7), (5.8).

(10.2) Definition. Salmon subalgebra. The subalgebra

$$
S_{2 m}(a, b, c)=\left(U_{2 m}(a, b, c), V_{2 m}(a, b, c)\right)
$$

is said to be Salmon subalgebra of $\left(S^{2 m}(W),[],\right)$. For the case $2 m=4$, we will call it as Salmon quartic subalgebra.

For a coordinate description of the subalgebra (with normalized coefficients of forms), we introduce the following numbers.

(10.3) Notation.

$$
B(m)=\frac{(2 m) !}{2(m !)(m !)}\left(=\frac{1}{2}\left(\begin{array}{c}
2 m \\
m
\end{array}\right)=(m+1) C(m) / 2\right),
$$

where $C(m)$ is the so-called $m$-th Catalan number.

(10.4) The coordinate description of Salmon subalgebra.

If $a=x_{0}, b=x_{1}, c=x_{2}$, then $U_{2 m}$ is the set of forms

$$
a_{0} x_{0}^{2 m}+a_{1} x_{1}^{2 m}+a_{2} x_{2}^{2 m}+2 B(m)\left(b_{0} x_{1}^{m} x_{2}^{m}+b_{1} x_{2}^{m} x_{0}^{m}+b_{2} x_{0}^{m} x_{1}^{m}\right),
$$


$V_{2 m}$ is the set of forms

$$
p_{0} u_{0}^{2 m}+p_{1} u_{1}^{2 m}+p_{2} u_{2}^{2 m}+2 B(m)\left(q_{0} u_{1}^{m} u_{2}^{m}+q_{1} u_{2}^{m} u_{0}^{m}+q_{2} u_{0}^{m} u_{1}^{m}\right) .
$$

At No. 298 of "Treatise" [25], considering the case $m=2$ (that is the case of plane quartics), G. Salmon observed that if $F$ is (10.5), then the contravariant $\sigma(F)$ is of the same form (more precisely, of (10.6)), that is $\sigma(F)$ belongs to $V_{2 m}$. Also, he wrote down a dependence of coefficients $p_{i}, q_{i}$ of $\sigma(F)$ from coefficients $a_{i}, b_{i}$ of $F$ explicitly. For arbitrary $m$, such a dependence is indicated below.

$$
\begin{gathered}
p_{0}=a_{1} a_{2}+(-1)^{m} B(m) b_{0}^{2}, \\
p_{1}=a_{2} a_{0}+(-1)^{m} B(m) b_{1}^{2}, \\
p_{2}=a_{0} a_{1}+(-1)^{m} B(m) b_{2}^{2}, \\
q_{0}=b_{1} b_{2}+(-1)^{m} a_{0} b_{0}, \\
q_{1}=b_{2} b_{0}+(-1)^{m} a_{1} b_{1}, \\
q_{2}=b_{0} b_{1}+(-1)^{m} a_{2} b_{2} .
\end{gathered}
$$

These formulas generalize formulas (6.1) for conics and Salmon's mentioned formulas for quartics.

(10.8) Notation. Consider the following pair of pencils

$$
\begin{gathered}
U_{2 m}^{\prime}(a, b, c)=\left(\lambda\left(a^{2 m}+b^{2 m}+c^{2 m}\right)+\mu\left((b c)^{m}+(c a)^{m}+(a b)^{m}\right)\right) \subset U_{2 m}(a, b, c), \\
V_{2 m}^{\prime}(a, b, c)=\left(\lambda\left([b, c]^{2 m}+[c, a]^{2 m}+[a, b]^{2 m}\right)\right. \\
\left.+\mu\left(([b, c][c, a])^{m}+([c, a][a, b])^{m}+([a, b][b, c])^{m}\right)\right) \subset S^{2 m}\left(W^{*}\right) .
\end{gathered}
$$

(10.9) Remark. For the case $m=2, a=x_{0}, b=x_{1}, c=x_{2}$, such a pencil $U_{4}^{\prime}$ was considered by A. Wiman. See formula (5) from [32] or page 536 of [31]. Also, the part $U_{4}^{\prime}=U_{4}^{\prime}\left(x_{0}, x_{1}, x_{2}\right)$ coincides with the so called FKT pencil in [23], as well as with the family of quartics considered in the postscript of Edge's paper [6].

(10.10) Theorem. $\left(U_{2 m}^{\prime}(a, b, c), V_{2 m}^{\prime}(a, b, c)\right)$ is a subalgebra of $\left(S^{2 m}(W),[],\right)$.

Proof. Also, it is a consequence of (5.7), (5.8).

(10.11) Definition. Wiman subalgebra. The pair

$$
W_{2 m}(a, b, c)=\left(U_{2 m}^{\prime}(a, b, c), V_{2 m}^{\prime}(a, b, c)\right)
$$

is said to be Wiman subalgebra of $\left(S^{2 m}(W),[],\right)$ or Wiman's idempotent pencil.

(10.12) Notation. $W_{2 m}=W_{2 m}\left(x_{0}, x_{1}, x_{2}\right)=\left(U_{2 m}^{\prime}, V_{2 m}^{\prime}\right)$.

(10.12) Theorem. Any Fermat's curve of even degree (that is form $F=$ $a^{n}+b^{n}+c^{n}$, where $n=2 m$ is even, $a, b, c$ are three linearly independent linear forms) is an idempotent in $S^{n}(W)$.

Proof. Indeed, according to (5.7), (5.8), we see that

$$
\begin{gathered}
\widehat{F}=[F, F] / 2=[a, b]^{n}+[a, c]^{n}+[b, c]^{n}, \\
\widehat{\widehat{F}}=<a . b . c>^{n}\left(a^{n}+b^{n}+c^{n}\right)=<a . b . c>^{n} F .
\end{gathered}
$$


(10.13) Theorem. If $k \neq m$, then form

$$
F=a^{2 m-k} b^{k}+b^{2 m-k} c^{k}+c^{2 m-k} a^{k}
$$

from $S^{2 m}(W)$ is an idempotent.

Proof. Because of (5.7), $\widehat{F}$ is proportional to

$$
[a, b]^{2 m-1}[b, c]^{k}+[b, c]^{2 m-k}[c, a]^{k}+[c, a]^{2 m-k}[a, b]^{k},
$$

therefore, using (5.7) and (5.8), we see that $\widehat{F}$ is proportional to $F$.

(10.14) Corollary. The Klein quartic $x_{0}^{3} x_{1}+x_{1}^{3} x_{2}+x_{2}^{3} x_{0}$ is an idempotent of the bialgebra $S^{4}(W)$.

(10.15) Theorem.

(1) The form

$$
\lambda\left(x_{0}^{4 l}+x_{1}^{4 l}+x_{2}^{4 l}\right)+2 B(2 l) \mu\left(\left(x_{1} x_{2}\right)^{2 l}+\left(x_{2} x_{0}\right)^{2 l}+\left(x_{0} x_{1}\right)^{2 l}\right)
$$

of Wiman pencil $W_{4 l}$ is an idempotent if only if one of the following conditions is fulfilled.

(i) $(\lambda: \mu)=(1: 0)$,

(ii) $(\lambda: \mu)=(B(2 l): 0)$,

(iii) $\quad 2 \lambda^{2}+2 \lambda \mu+(B(2 l)+1) \mu^{2}=0, \quad(\lambda, \mu) \neq(0,0)$.

(2) The form

$$
\begin{gathered}
\lambda\left(x_{0}^{4 l+2}+x_{1}^{4 l+2}+x_{2}^{4 l+2}\right) \\
+2 B(2 l+1) \mu\left(\left(x_{1} x_{2}\right)^{2 l+1}+\left(x_{2} x_{0}\right)^{2 l+1}+\left(x_{0} x_{1}\right)^{2 l+1}\right)
\end{gathered}
$$

of Wiman pencil $W_{4 l+2}$ is an idempotent if only if one of the following conditions is fulfilled.

(i) $(\lambda: \mu)=(1: 0)$,

(ii) $\quad 2 \lambda^{2}-\lambda \mu-B(2 l+1) \mu^{2}=0, \quad(\lambda, \mu) \neq(0,0)$.

Proof. It is an easy consequence of formulas (10.7).

(10.18) Example. In the quartic pencil $U_{4}^{\prime}$, idempotents are: a double conic, Fermat's quartic, and the quartics

$$
\begin{aligned}
& m\left(x_{0}^{4}+x_{1}^{4}+x_{2}^{4}\right)-6\left(x_{1}^{2} x_{2}^{2}+x_{2}^{2} x_{0}^{2}+x_{0}^{2} x_{1}^{2}\right), \\
& \bar{m}\left(x_{0}^{4}+x_{1}^{4}+x_{2}^{4}\right)-6\left(x_{1}^{2} x_{2}^{2}+x_{2}^{2} x_{0}^{2}+x_{0}^{2} x_{1}^{2}\right),
\end{aligned}
$$

where

$$
m=\frac{-1+i \sqrt{7}}{2}, \quad \bar{m}=\frac{-1-i \sqrt{7}}{2} .
$$

Two last quartics are projectively equivalent to Klein's quartic, but here we do not write down the formulas for a projective transformation, projective coincidence of these two quartics with Klein's one was indicated by Wiman [31], page 536, the equivalence was used by Dolgachev and Kanev [5], Rodríguez and González-Aguilera [23].

(10.19) Theorem. A quartic is an idempotent if and only if it is either a double conic or a Fermat quartic or a Klein quartic. 
Proof. This theorem is a reformulation of Edgardo Ciani's theorem [4], in his formulation, Fermat's quartic was missed, because, instead of the iteration of $\sigma$, he considered the Clebsch covariant (see (6.6)) vanishing at Fermat's quartics.

(10.20) Remark. Clebsch quartics of the Wiman quartic pencil. Let us consider the catalecticant $J(\lambda, \mu)$ of curves from $U_{4}^{\prime}$. It is not hard to calculate that

$$
J(\lambda, \mu)=\mu^{3}(\lambda-\mu)^{2}(\lambda+2 \mu) .
$$

It is well-known, that if the catalecticant of a quartic vanishes, then the corresponding form (the so called Clebsch quartic) is a sum of five (or less ) fourth powers of ternary linear forms (see definition (6.12.1) from [5]). In our case, factor of multiplicity $m$ of the catalecticant $J(\lambda, \mu)$ corresponds to a Lüroth quartic representable as a sum of $(6-m)$ powers of linear forms.

Indeed, the form corresponding to the first factor $\mu^{3}$ defines Fermat's quartic and the quartic is a sum of three powers of linear forms. Further, the form corresponding to the second factor $(\lambda-\mu)^{2}$ is proportional to the following sum of four powers of linear forms

$$
\left(x_{0}+x_{1}+x_{2}\right)^{4}+\left(-x_{0}+x_{1}+x_{2}\right)^{4}+\left(x_{0}-x_{1}+x_{2}\right)^{4}+\left(x_{0}+x_{1}-x_{2}\right)^{4} .
$$

It is a special Capolari quartic (see definition (6.11) from [5]).

The third factor $(\lambda+2 \mu)$ corresponds to form

$$
4\left(x_{0}^{4}+x_{1}^{4}+x_{2}^{4}\right)-12\left(x_{1}^{2} x_{2}^{2}+x_{2}^{2} x_{0}^{2}+x_{0}^{2} x_{1}^{2}\right),
$$

the last form belongs to the following family of Clebsch-Lüroth quartics

$$
\left(k x_{0}+x_{1}+x_{2}\right)^{4}+\left(k x_{0}-x_{1}-x_{2}\right)^{4}+\left(k x_{0}+x_{1}-x_{2}\right)^{4}+\left(k x_{0}-x_{1}+x_{2}\right)^{4}+l x_{0}^{4} \text {. }
$$

The quartic with $k=i \sqrt{2}, l=-4$ coincides with the form.

(10.21) Remark. Other catalecticants. The catalecticant for the form (10.16) is the following product belonging to $\mathbb{Q}[\lambda, \mu]$ :

$$
\mu^{3}\left(\lambda^{3}+a \lambda^{2} \mu+b \lambda \mu^{2}+c \mu^{3}\right),
$$

the catalecticant for the form (10.17) is the following product belonging to $\mathbb{Q}[\lambda, \mu]$ :

$$
\mu^{4}\left(\lambda^{2}+a \lambda \mu+b \mu^{2}\right)
$$

where $a, b, c$ are some rational constants.

(10.22) Remark. Plücker's form of a quartic is $A^{2}+a b c d$, where $A$ is quadratic form, $a, b, c, d$ are linear forms, these linear forms define four bitangents of the quartic. One can reduce quartics of Wiman pencil $U_{4}^{\prime}$ to Plücker's form. If we take the substitution

$$
x_{0}=y_{1}+y_{2}, x_{1}=y_{2}+y_{0}, x_{2}=y_{0}+y_{1}
$$

indicated by Edge [6], then we get quartics

$$
((\lambda+3 \mu) / 2)\left(y_{0}^{2}+y_{1}^{2}+y_{2}^{2}+\left(y_{0}+y_{1}+y_{2}\right)^{2}\right)^{2}+8(3 \mu-\lambda) y_{0} y_{1} y_{2}\left(y_{0}+y_{1}+y_{2}\right) .
$$

By the way, we see four evident bitangents to all the quartics from Wiman's pencil $U_{4}^{\prime}$, the bitangents were indicated in a picture (more precisely, Figure 5) of [23]. The equations of these special four bitangents are those in [6], which do not contain additional "irrationalities". 
Especially, Klein's quartic has a natural Plücker's form. Klein and Brioschi proved it, but their ways were longer, because they started from the initial form (10.14) of Klein's quartic. See [2], [18].

(10.23) Remark. Syzygetic and equianharmonic quadruples of bitangents.

According to Frobenius and Weber (see Weber, [29] §116), four bitangents of a plane quartic are syzygetic, if eight points of tangency are located on a common conic. A generic quartic has 315 sets of four syzygetic bitangents. We say that syzygetic quadruple of bitangents is equianharmonic, if for every of the bitangents, each of two points of contact together with the three intersection points of the bitangent with three other bitangents constitute an equianharmonic point quadruple (see definition in $(8.2)$ ).

The four bitangents $y_{0}, y_{1}, y_{2}, y_{0}+y_{1}+y_{2}$ from (10.22) are syzygetic and equianharmonic. Fermat's or Klein's quartics have several (at least more than one) syzygetic and equianharmonic bitangent quadruples: it is a consequence of having a rich group of projective automorphisms. A generic curve from Wiman quartic pencil has a unique such a quadruple.

It is a characterization of nonsingular quartic idempotents and quartic idempotent pencils.

Note that Wiman's quartic pencil $U_{4}^{\prime}$ contains a singular nodal quartic, that is the lemniscate (8.9). Any rational nodal quartic has four bitangents exactly, and, of course, for the lemniscate these four bitangents are syzygetic and equianharmonic.

(10.24) Remark. A list of all 28 bitangents for a generic curve of Wiman's pencil $U_{4}^{\prime}$ is described by W.L. Edge in [6], No.14 (Postscript). The equations of these bitangents include an irrational quantity $\gamma$ such that in (10.16) (where $l=2$ and $B(4)=6$ )

$$
(\lambda: \mu)=\left(3\left(1+\gamma^{2}\right)^{2}:-\left(1+\gamma^{4}\right)\right) .
$$

Edge [6] gave the list and also symmetric determinantal representations of Wiman's curves (Edge's symmetric matrices have zero diagonal). Also, he describes a way of enumeration of syzygetic sets of bitangents of the curve, this combinatorial way is based on Cayley's combinatorial symbolism, this symbolism is described by Weber [29] also.

(10.25) Theorem. Salmon quartic algebra is semisimple.

Proof. We will use the coordinate description of Salmon quartic algebra, that is elements of $U_{4}$ we will present as

$$
a_{0} x_{0}^{4}+a_{1} x_{1}^{4}+a_{2} x_{2}^{4}+6\left(b_{0} x_{1}^{2} x_{2}^{2}+b_{1} x_{2}^{2} x_{0}^{2}+b_{2} x_{0}^{2} x_{1}^{2}\right) .
$$

Then one can write down six mutually apolar idempotent quartics. Here they are.

$$
\begin{gathered}
Q_{1}=x_{0}^{4}+\omega x_{1}^{4}+\omega^{2} x_{2}^{4}, \\
Q_{2}=x_{0}^{4}+\omega^{2} x_{1}^{4}+\omega x_{2}^{4}, \\
Q_{3}=m\left(x_{0}^{4}+x_{1}^{4}+x_{2}^{4}\right)-6\left(x_{1}^{2} x_{2}^{2}+x_{2}^{2} x_{0}^{2}+x_{0}^{2} x_{1}^{2}\right), \\
Q_{4}=m\left(x_{0}^{4}+x_{1}^{4}+x_{2}^{4}\right)+6\left(-x_{1}^{2} x_{2}^{2}+x_{2}^{2} x_{0}^{2}+x_{0}^{2} x_{1}^{2}\right), \\
Q_{5}=m\left(x_{0}^{4}+x_{1}^{4}+x_{2}^{4}\right)+6\left(x_{1}^{2} x_{2}^{2}-x_{2}^{2} x_{0}^{2}+x_{0}^{2} x_{1}^{2}\right), \\
Q_{6}=m\left(x_{0}^{4}+x_{1}^{4}+x_{2}^{4}\right)+6\left(x_{1}^{2} x_{2}^{2}+x_{2}^{2} x_{0}^{2}-x_{0}^{2} x_{1}^{2}\right),
\end{gathered}
$$


where $m$ is the number from (10.18). The reciprocal quartics are

$$
\begin{gathered}
\widehat{Q_{1}}=R_{1}=u_{0}^{4}+\omega^{2} u_{1}^{4}+\omega u_{2}^{4}, \\
\widehat{Q_{2}}=R_{2}=u_{0}^{4}+\omega u_{1}^{4}+\omega^{2} u_{2}^{4}, \\
\widehat{Q_{3}}=R_{3}=(1+m)\left(u_{0}^{4}+u_{1}^{4}+u_{2}^{4}\right)+6(1-m)\left(u_{1}^{2} u_{2}^{2}+u_{2}^{2} u_{0}^{2}+u_{0}^{2} u_{1}^{2}\right), \\
\widehat{Q_{4}}=R_{4}=(1+m)\left(u_{0}^{4}+u_{1}^{4}+u_{2}^{4}\right)+6(1-m)\left(u_{1}^{2} u_{2}^{2}-u_{2}^{2} u_{0}^{2}-u_{0}^{2} u_{1}^{2}\right), \\
\widehat{Q_{5}}=R_{5}=(1+m)\left(u_{0}^{4}+u_{1}^{4}+u_{2}^{4}\right)+6(1-m)\left(-u_{1}^{2} u_{2}^{2}+u_{2}^{2} u_{0}^{2}-u_{0}^{2} u_{1}^{2}\right), \\
\widehat{Q_{6}}=R_{6}=(1+m)\left(u_{0}^{4}+u_{1}^{4}+u_{2}^{4}\right)+6(1-m)\left(-u_{1}^{2} u_{2}^{2}-u_{2}^{2} u_{0}^{2}+u_{0}^{2} u_{1}^{2}\right) .
\end{gathered}
$$

The first two forms $Q_{1}, Q_{2}$ are Fermat quartics, other four forms $Q_{3}, Q_{4}, Q_{5}, Q_{6}$ are Klein quartics.

(10.26) Remark. We can add six Klein's quartics

$$
\begin{gathered}
Q_{7}=x_{0}^{3} x_{1}+x_{1}^{3} x_{2}+x_{2}^{3} x_{0}, \\
Q_{8}=x_{0}^{3} x_{1}+\omega x_{1}^{3} x_{2}+\omega^{2} x_{2}^{3} x_{0}, \\
Q_{9}=x_{0}^{3} x_{1}+\omega^{2} x_{1}^{3} x_{2}+\omega x_{2}^{3} x_{0}, \\
Q_{10}=x_{0}^{3} x_{2}+x_{2}^{3} x_{1}+x_{1}^{3} x_{0}, \\
Q_{11}=x_{0}^{3} x_{2}+\omega x_{2}^{3} x_{1}+\omega^{2} x_{1}^{3} x_{0}, \\
Q_{12}=x_{0}^{3} x_{2}+\omega^{2} x_{2}^{3} x_{1}+\omega x_{1}^{3} x_{0}
\end{gathered}
$$

and get 12 mutually apolar idempotents $Q_{1}, \ldots, Q_{12}$, but $12<15$, therefore it does not prove semisimplicity of the bialgebra of quartics.

11. Conic constructions of Salmon and Wiman quartic subalgebras. Our next goal is a description of Salmon quartic subalgebra with the help of a pair of ternary quadratic forms.

(11.1) Notation. Let $A, B$ be two linearly independent nondegenerate ternary quadratic forms. We denote by $L(A, B)$ (resp. $M(A, B)$ ) the vector subspace of the quartic space generated by

$$
A B, \quad A^{2}, \quad B^{2}, \quad A[\widehat{A}, \widehat{B}], \quad B[\widehat{A}, \widehat{B}], \quad[\widehat{A}, \widehat{B}]^{2}
$$

$$
\text { (resp. } \left.\widehat{A}^{2}, \widehat{B}^{2}, \widehat{A} \cdot \widehat{B}, \widehat{A} \cdot[A, B], \widehat{B} \cdot[A, B], \quad[A, B]^{2}\right) .
$$

Another notation: $S(A, B)=(L(A, B), M(A, B))$.

(11.4) Theorem.

(1) $\operatorname{dim} L(A, B)=6$ if and only if $A$ does not tangent $B$.

(2) $\operatorname{dim} M(A, B)=6$ if and only if $A$ does not tangent $B$.

(3) If $a, b, c$ are linearly independent ternary linear forms,

$$
A=a^{2}+b^{2}+c^{2}, \quad B=k a^{2}+l b^{2}+m c^{2},
$$

where $k \neq l, k \neq m, \quad l \neq m$, then

$$
(L(A, B), M(A, B))=\left(U_{4}(a, b, c), V_{4}(a, b, c)\right)
$$

(according to Notations (10.1)). 
Proof. If we suppose that

$$
A=x_{0}^{2}+x_{1}^{2}+x_{2}^{2}, B=k x_{0}^{2}+l x_{1}^{2}+m x_{2}^{2},
$$

then the determinant of the matrix expressing forms (11.2) in terms of monomials

$$
x_{0}^{4}, \quad x_{1}^{4}, \quad x_{2}^{4}, \quad x_{1}^{2} x_{2}^{2}, \quad x_{2}^{2} x_{0}^{2}, \quad x_{0}^{2} x_{1}^{2},
$$

is equal to

$$
((k-l)(k-m)(l-m))^{4} .
$$

This proves (1). Proof of (2) is similar.

(11.5) Corollary. If $A \perp B$, then the six quartics (11.2) (as well as six quartics (11.3)) are linearly independent in the vector space of ternary quartics.

(11.6) Remark. The theory of invariants (according to G. Salmon's "Conic Sections" [24], No. 372) gives the following condition of mutual tangency of two conics $A=0, B=0$. It is vanishing of the discriminant of the (discriminant) binary form of variables $(t, s)$

$$
\operatorname{disc}(t A+s B)=\operatorname{disc}(A) t^{3}+<\widehat{A}, B>t^{3} s+<A, \widehat{B}>t s^{2}+\operatorname{disc}(B) s^{3},
$$

that is the condition is the following equality

$$
\begin{gathered}
18 \operatorname{disc}(A) \operatorname{disc}(B)<\widehat{A}, B><A, \widehat{B}>+<\widehat{A}, B>^{2}<\widehat{B}, A>^{2} \\
=27 \operatorname{disc}(A)^{2} \operatorname{disc}(B)^{2}+4 \operatorname{disc}(A)<\widehat{B}, A>^{3}+4 \operatorname{disc}(B)<\widehat{A}, B>^{3} .
\end{gathered}
$$

Of course, the dimensions of spaces $L(A, B), M(A, B)$ depend on the Segre symbol of the conic pencil $\lambda A+\mu B=0$. About the Segre symbol, one can see Hodge and Pedoe, [17], vol. II, pages 304-305, moreover possible types of pencils are described with pictures at pages 158-161 of book [26] by Semple and Kneebone. For example, if $A$ and $B$ have two points of intersection and they tangent at both the points (that is the corresponding Segre symbol is $[(1,1), 1]$ according to [16]), then $A, B$ generate a three-dimensional subalgebra: $\operatorname{dim} L(A, B)=\operatorname{dim} M(A, B)=3$.

(11.7) Theorem. For two non-degenerated conics $A, B$, and for a non-degenerate matrix

$$
\left(\begin{array}{ll}
a & b \\
c & d
\end{array}\right)
$$

we have

$$
L(a A+b B, c A+d B)=L(A, B), \quad M(a A+b B, c A+d B)=M(A, B) .
$$

Proof. It is enough to prove that $L(A, B+k A)=L(A, B)$, that is

$$
[\widehat{A}, \widehat{B+k} A] \in L(A, B) \text {. }
$$

Because of the bilinear property of the bimultiplication and of (6.7),

$$
[\widehat{A}, \widehat{B+k A}]=\left[\widehat{A}, \widehat{B}+k[A, B]+k^{2} \widehat{A}\right]=[\widehat{A}, \widehat{B}]+k[\widehat{A},[A, B]]+k^{2} \widehat{\widehat{A}} .
$$

Moreover, according to (6.9), (6.4),

$$
[\widehat{A},[A, B]]=\operatorname{disc}(A) B+<\widehat{A}, B>B, \quad \widehat{\widehat{A}}=\operatorname{disc}(A) A .
$$


(11.8) Theorem. For every Salmon subalgebra $S(A, B)$, one can find a pair of conics $C, D$ such that

$$
C \perp D, \quad L(A, B)=L(C, D), \quad M(A, B)=M(C, D) .
$$

In the conic pencil $(\lambda A+\mu B)$, such a pair is unique (up to interchange and proportionality), but in the subalgebra the family of such pairs is a variety of dimension 2 at least.

Proof. It is clear, if we consider a common self-polar triangle for $A$ and $B$.

(11.9) Notation. Let $A, B$ be two mutually apolar nondegenerate ternary quadratic forms. Let

$$
L_{0}(A, B)=K \cdot A B+K \cdot[\widehat{A}, \widehat{B}]^{2} \subset L(A, B)
$$

denote the vector space spanned by $A B,[\widehat{A}, \widehat{B}]^{2}$,

$$
M_{0}(A, B)=K \cdot \widehat{A} \cdot \widehat{B}+K \cdot[A, B]^{2} \subset M(A, B)
$$

denote the vector space spanned by $\widehat{A} \cdot \widehat{B},[A, B]^{2}$.

Both the spaces are two-dimensional, because $A, B$ are nondegenerate and nonproportional.

Another notation. $W(A, B)=\left(L_{0}(A, B), M_{0}(A, B)\right)$.

(11.10) Theorem. (1) $\left(L_{0}(A, B), M_{0}(A, B)\right)$ is a subalgebra.

(2) If as in (7.8),

$$
A=x_{0}^{2}+\omega x_{1}^{2}+\omega^{2} x_{2}^{2}, \quad B=x_{0}^{2}+\omega^{2} x_{1}^{2}+\omega x_{2}^{2},
$$

then $\left(L_{0}(A, B), M_{0}(A, B)\right)=W_{4}$

Proof. It is enough to use the particular case indicated in (7.8), but, because of further necessity of some details, we will give a proof without considering the particular case. We will prove that if $A \perp B$, $\operatorname{disc}(A) \cdot \operatorname{disc}(B)=\delta$, and

$$
F=x \cdot A B+y \cdot \delta^{-1}[\widehat{A}, \widehat{B}]^{2},
$$

then $\sigma(F) \in M_{0}(A, B)$, and we will find an explicit expression for $\sigma(F)$. According to $(2.6)$,

$$
\sigma(F)=x^{2} \sigma(A B)+y^{2} \delta^{-2}\left(\sigma[\widehat{A}, \widehat{B}]^{2}\right)+x y \delta^{-1}\left[A B,[\widehat{A}, \widehat{B}]^{2}\right],
$$

Further, according to (9.14), (9.12) and (6.4),

$$
\begin{gathered}
\sigma(F)=x^{2}\left([A, B]^{2} / 12+[\widehat{A}, \widehat{B}]\right)+y^{2} \delta^{-2}(\widehat{(\widehat{A}, \widehat{B}]})^{2} \\
\left.+x y \delta^{-1}([A,[\widehat{A}, \widehat{B}]] \cdot[B,[\widehat{A}, \widehat{B}]]-(2 / 3)[A, B] \cdot \widehat{[\widehat{A}, \widehat{B}}]\right) .
\end{gathered}
$$

But because of mutual apolarity of $A, B$ and in virtue of $(6.9),(6,17)$, we have

$$
\delta^{-1}\left([A,[\widehat{A}, \widehat{B}]] \cdot[B,[\widehat{A}, \widehat{B}]]=A B, \quad \delta^{-1} \widehat{[\widehat{A}, \widehat{B}]}=-[A, B]\right.
$$

Therefore

$$
\sigma(F)=\left(x^{2}+x y\right) \widehat{A} \cdot \widehat{B}+\frac{(x+4 y)^{2}}{12}[A, B]^{2} .
$$

Now, we see that the form $\sigma(F)$ belongs to $M_{0}(A, B)$ and have useful description (11.11) of the form. 
(11.12) Definition. If $A, B$ are two conics with four distinct points of intersection, then we will say that $(L(A, B), M(A, B))$ is Salmon subalgebra generated by $A, B$. If moreover $A \perp B$, then we say that $\left(L_{0}(A, B), M_{0}(A, B)\right)$ is Wiman subalgebra of the Salmon subalgebra $(L(A, B), M(A, B))$.

(11.13) Remark. Any Wiman quartic subalgebra defines a collection of simple geometric objects:

(i) two mutually apolar conics,

(ii) four points of intersection of the conics,

(iii) four common tangents of the two mutually apolar conics (union of these four lines is a curve of the corresponding Wiman pencil,

(iv) the Bernoulli lemniscate.

For the case of canonical coordinates (7.9), equations of the conics are given in (7.8), the equation of the lemniscate is (8.9), four lines are indicated in (10.22), [6], [23]. Here, we would like to show that each of the objects determines Wiman subalgebra.

(i) Two mutually apolar conics determine a Wiman subalgebra according to (11.10).

(ii) Four points define a pencil of conics, the pencil contains exactly two mutually apolar conics, the conics define a Wiman subalgebra.

(iii) Because of the projective duality, it enough to refer on (ii), but we would like to describe shortly Klein's and Brioschi's approach (hidden in . [18] and [2]). It is enough to construct two Klein's quartics of the pencil, they would be basic curves of Wiman pencil. In [18], §4, formula (12) Klein writes equation of his curve in the form

$$
\left(z_{1}^{2}+z_{2}^{2}+z_{3}^{2}+z_{4}^{2}\right)^{2}-2 \Lambda z_{1} z_{2} z_{3} z_{4}=0,
$$

where

$$
\Lambda=7+3 \sqrt{-7}, \text { or } \Lambda=7-3 \sqrt{-7},
$$

$z_{i}$ are four linear forms whose sum is zero, their mixed products are equal one to another (up to a sign, of course), more precisely,

$$
<z_{i} \cdot z_{j} \cdot z_{k}>= \pm 3 \cdot(56)^{2} \cdot \lambda \cdot\left(\omega^{2}-\omega\right) .
$$

(Brioschi [2] hides $\sqrt{\Lambda}$ under the left square of the curve equation).

Let $a, b, c, d$ be four linear forms, no three of which are linearly dependent. We define a quadratic form $M(a, b, c, d)$ depending from these ternary linear forms $a, b, c, d$ :

$$
\left.M=M(a, b, c, d)=a^{2}<b c d>^{2}+b^{2}<a c d>^{2}+c^{2}<a b d>^{2}+d^{2}<a b c\right\rangle^{2} .
$$

Note that $M$ is a sum of squares of four linear forms, whose sum is equal to zero (see (5.3)). The geometric meaning of $M$ is as follows. The conic $M=0$ intersects every line $a=0, b=0, c=0, d=0$ equianharmonically (see definition in (8.2)) with respect to three points of intersection of the line with three other lines (of course, all the eight equianharmonic points belong to a common conic). Note that this conic is irreducible. The pencil $\left(\lambda M^{2}+\mu a b c d\right)$ is a Wiman pencil (containing of course two Klein's quartics, according to Klein and Brioschi). More precisely, the quartic $\sigma(a b c d)$ is decomposable (see Example (8.2)):

$$
\sigma(a b c d)=k P Q
$$


where $P\left(u_{0}, u_{1}, u_{2}\right), Q\left(u_{0}, u_{1}, u_{2}\right)$ are two mutually apolar quadratic forms, $k \in K$, $k \neq 0$. Then

$$
K[P, Q]^{2}+K \widehat{P} \widehat{Q}=\left(\lambda M^{2}+\mu a b c d\right),
$$

moreover

$$
K[\widehat{P}, \widehat{Q}]^{2}+K P Q=\left(\lambda\left(p^{2}+q^{2}+r^{2}+s^{2}\right)^{2}+\mu p q r s\right),
$$

where $p, q, r, s$ are four bitangents for conics $P=0, Q=0$ (more explicit calculations for dual situation are presented in (7.15) and (13.7) in terms of the canonical coordinates (7.9)).

(iv) Producing a Wiman subalgebra from the Bernoulli lemniscate. Let $F=0$ be an equation of the lemniscate $L$. First of all, any rational quartic with three nodal points has exactly four bitangents. Let $P$ be a quadratic form such that $\sigma(F)=P^{2}$ (see Example (8.7)). Then $\left(\lambda F+\mu \widehat{P}^{2}\right)$ is a Wiman pencil. More precisely, if $a, b, c, d$ are four bitangents of the lemniscate, then eight points

$$
(a \cap L) \cup(b \cap L) \cup(c \cap L) \cup(d \cap L)
$$

belong to a common conic $C$, this conic is $M(a, b, c, d)=0$, and one can see coincidence of two pencils:

$$
\left(\lambda F+\mu \widehat{P}^{2}\right)=(\lambda M+\mu a b c d) .
$$

(11.14) Remark. If $A, B$ are two mutually apolar nondegenerate ternary quadratic forms, $\operatorname{disc}(A)=d(A), \operatorname{disc}(B)=d(B),(\lambda, \mu) \neq(0,0)$, then quartic

$$
\lambda A B+\mu(d(A) d(B))^{-1}[\widehat{A}, \widehat{B}]^{2} \in L_{0}(A, B)
$$

is

(i) a double conic for $\lambda=0$,

(ii) a Bernoulli lemniscate for $\lambda=-\mu$,

(iii) a Fermat quartic for $\lambda=2 \mu$,

(iv) a Klein quartic for

$$
\lambda=\frac{-35 \pm 9 \sqrt{-7}}{32} \mu
$$

(that is $16 \lambda^{2}+35 \lambda \mu+28 \mu^{2}=0$ ).

(11.15) Theorem. If $A, B, C$ are three mutually apolar ternary quadratic forms belonging to a sextuple of mutually apolar forms, then Wiman subalgebras $W(A, B)$ and $W(A, C)$ are mutually apolar in the sense that

$$
<L_{0}(A, B), M_{0}(A, C)>=\{0\},<L_{0}(A, C), M_{0}(A, B)>=\{0\} .
$$

Proof. The relations of orthogonality are consequences of Theorem (7.13). Indeed we have to prove that

$$
<A B, \widehat{A} \widehat{C}>=0, \quad<A B,[A, C]^{2}>=0, \quad<[A, B]^{2}, \quad[\widehat{A}, \widehat{C}]^{2}>=0 .
$$

First of them follows from (9.4) and (7.13), the second identity is a consequence of (7.13), (9.2), (6.17), the third one follows from (7.13). (9.1), (6.17).

(11.16) Theorem. Let $A, B, C, D$ be four distinct conics from Gerbaldi's six,

$$
d=\operatorname{disc}(A)\left(=\operatorname{disc}(B)=\operatorname{disc}(C)=\operatorname{disc}(D)=d=-c^{2}\right),
$$

where $c$ is the constant from the equations of Gerbaldi's conics (7.11). 
(1) For the following two quartics

$$
\begin{aligned}
& E^{\prime}(y)=A B+y d^{-2}[\widehat{A}, \widehat{B}]^{2} \in L_{0}(A, B) \\
& E^{\prime \prime}(y)=C D+y d^{-2}[\widehat{C}, \widehat{D}]^{2} \in L_{0}(C, D)
\end{aligned}
$$

the relation

$$
<E^{\prime}(y), \sigma\left(E^{\prime \prime}(y)\right)>=0
$$

is fulfilled if and only if $y$ is a root of cubic equation

$$
c^{2}\left(16 y^{3}-69 y^{2}-132 y-20\right)+9(2 y-1)^{2}=0 .
$$

Proof. If $A, B, C, D$ are four distinct mutually apolar conics,

$$
\begin{gathered}
\operatorname{disc}(A)=\operatorname{disc}(B)=d, \\
F=x[A, B]+y d^{-2}[\widehat{A}, \widehat{B}]^{2} \in L_{0}(A, B), \\
G=\xi \widehat{C} \widehat{D}+\eta[C, D]^{2} \in M_{0}(C, D),
\end{gathered}
$$

then

$$
\begin{gathered}
<F, G>=\frac{1}{3}<[A, B],[\widehat{C}, \widehat{D}]>(-x \xi+2 x \eta+\operatorname{disc}(A) \operatorname{disc}(B)(2 y \xi-4 y \eta)) \\
+y \eta<[A, B],[\widehat{C}, \widehat{D}]>^{2}+x \eta<A . C . D><B . C . D>+y \xi<\widehat{A} . \widehat{B} . \widehat{C}><\widehat{A} \cdot \widehat{B} . \widehat{D}>.
\end{gathered}
$$

Further, if $F=E^{\prime}(y), G=\sigma\left(E^{\prime \prime}(y)\right)$, then

$$
x=1, \xi=1+y, \eta=(1+4 y)^{2} / 12
$$

(see (11.11)).

Now, using Theorem (7.14), it is not hard to see that $\left\langle E^{\prime}(y), \sigma\left(E^{\prime \prime}(y)\right)\right\rangle$ is proportional to the left hand side of (11.17).

(11.18) Remark. The substitution

$$
y=(1-3 t) / 2
$$

simplifies equation (11.17):

$$
8 t^{3}+6(c+4) t^{2}-42 t+15=0 .
$$

The discriminant $D$ of the latter cubic polynomial, more precisely, of the corresponding binary form

$$
8 t_{0}^{3}+3 \cdot 2(c+4) t_{0}^{2} t_{1}+3 \cdot(-14) t_{0} t_{1}^{2}+15 t_{1}^{3}
$$

is presented by

$$
D=2^{7}(201 c-26)=2^{5}(97+201 i \sqrt{15})
$$

the discriminant is not a square in the field $\mathbb{Q}(c)$.

If one would like to indicate an equation with rational coefficients for a root $t$ of equation (11.19), then we can write it down. This root is a root of the equation

$$
64 t^{6}+408 t^{5}+12 t^{4}-1902 t^{3}+2529 t^{2}-1260 t+225=0
$$


The last equation has a solvable Galois group over $\mathbb{Q}$, because we saw a reduction of the equation to the cubic equation (11.19) over the quadratic field $\mathbb{Q}(\sqrt{-15})$. The discriminant $D_{1}$ of the sextic polynomial (11.21) has the following factorization

$$
D_{1}=2^{18} \times 3^{19} \times 5^{7} \times 601 .
$$

We add that $601=3^{6}-2^{7}=5^{4}-5^{2}+1$. Also, the last factor arises by factorization of the norm of the number $D$ from (11.19):

$$
\operatorname{Norm}\left(2^{-4} D\right)=\operatorname{Norm}(2(201 c-26))=153856=2^{8} \cdot 601 .
$$

It is a curio that

$$
\operatorname{Norm}(2(201 c+26))=2^{7} \times 5 \times 17 \times 257=2^{7}\left(2^{2^{1}}+1\right)\left(2^{2^{2}}+1\right)\left(2^{2^{3}}+1\right) .
$$

(11.22) Example. A four-dimensional subalgebra of Salmon algebra. Suppose that quadratic forms $A, B$ generating a Salmon algebra are normalized in such a way that their discriminants are equal: $\operatorname{disc}(A)=\operatorname{disc}(B)$. In this case, the pair $\left(L_{1}(A, B), M_{1}(A, B)\right)$, where $L_{1}(A, B)$ is spanned by

$$
A B, A^{2}+B^{2},(A+B)[\widehat{A}, \widehat{B}],[\widehat{A}, \widehat{B}]^{2},
$$

$M_{1}(A, B)$ is spanned by

$$
\widehat{A}^{2}+\widehat{B}^{2}, \widehat{A} \cdot \widehat{B}, \quad(\widehat{A}+\widehat{B}) \cdot[A, B], \quad[A, B]^{2},
$$

is a subalgebra of Salmon's algebra. We will not need this subalgebra.

12. Proofs of theorems (1.9), (1.10), (1.11).

(12.1) Proof of (1.9). A preliminary remark. The fifteen idempotent pencils will be $W\left(f_{i}, f_{j}\right), i \neq j, i, j \in\{1,2,3,4,5,6\}$ (see Notation (11.9), Theorem (11.10), Definition (11.12)). That is for formation of the cogredient parts

$$
L_{0}\left(f_{i}, f_{j}\right)=\left(\lambda f_{i} f_{j}+\mu\left[\widehat{f}_{i}, \widehat{f}_{j}\right]^{2}\right)
$$

of the pencils, we mix the bases (7.14)(i) and (7.14)(iii). A similar process goes by formation of contragredient parts. Formally, we do not need Theorem (7.14), but it seems that the heart of the matter of the weak idempotency is hidden in the big matrix from the proof of (7.14), because the minimal polynomial of the matrix is quadratic, that is the matrix is an idempotent of level 1 in some sense (or in the sense of Definition (1.6) applied to the matrix algebra).

Let us fix a root $y$ of equation (11.17). Then quartics

$$
E_{i j}=f_{i} f_{j}+y c^{-4}\left[\widehat{f}_{i}, \widehat{f}_{j}\right]
$$

and their reciprocals $\sigma\left(E_{i j}\right)$ constitute two mutually reciprocal bases according to Theorems (11.15), (11.16). Quartics

$$
P_{i j}=\widehat{f}_{i} \widehat{f}_{j}+y c^{4}\left[f_{i}, f_{j}\right]
$$

have similar properties.

(12.2) Proof of (1.10). The fifteen semisimple (according to (10.25)) subalgebras are $S\left(f_{i}, f_{j}\right) \quad(i \neq j), i, j \in\{1,2,3,4,5,6\}$ (see Notation (11.1), Theorem (11.4)). These Salmon subalgebras contain Wiman subalgebras from (12.1), therefore we may take the bases from (12.1). 
(12.3) Proof of (1.11). It is necessary to prove that if $A_{1}, \ldots, A_{6}$ are six mutually apolar conics, then the union of the base loci for Wiman pencils $L_{0}\left(A_{i}, A_{j}\right)$ (see Notation (11.9)) is the union $U$ of points of intersection of the conics. If $A, B$ are two mutually apolar conics, then the base locus of $L_{0}(A, B)$ consists of eight points of reciprocal contact of the pencil of quartics ( these eight points are the points of contact of four bitangents for $A, B)$. Indeed, for the case of canonical coordinates, it was indicated by R. Rodríguez and V.González-Aguilera in [23] (page 53 and Figure 5 of page 54), see also Remarks (10.22), (10.23), (11.13).

Now we may apply Theorem (7.15).

The theorems are proved.

\section{The bialgebra of ternary sextics.}

(13.1) Formulas for the reciprocal of a ternary sextic. A ternary sextic has 28 coefficients. Its full description is

$$
\begin{gathered}
F\left(x_{0}, x_{1}, x_{2}\right)=\sum a(\text { ijklmn }) x_{i} x_{j} x_{k} x_{l} x_{m} x_{n}= \\
a(000000) x_{0}^{6}+a(111111) x_{1}^{6}+a(222222) x_{2}^{6} \\
+6 a(000001) x_{0}^{5} x_{1}+6 a(000002) x_{0}^{5} x_{2}+6 a(111110) x_{1}^{5} x_{0} \\
+6 a(111112) x_{1}^{5} x_{2}+6 a(222220) x_{2}^{5} x_{0}+6 a(222221) x_{2}^{5} x_{1} \\
+15 a(000011) x_{0}^{4} x_{1}^{2}+15 a(000022) x_{0}^{4} x_{2}^{2}+15 a(111100) x_{1}^{4} x_{0}^{2} \\
+15 a(111122) x_{1}^{4} x_{2}^{2}+15 a(222200) x_{2}^{4} x_{0}^{2}+15 a(222211) x_{2}^{4} x_{1}^{2} \\
+20 a(000111) x_{0}^{3} x_{1}^{3}+20 a(111222) x_{1}^{3} x_{2}^{3}+20 a(222000) x_{2}^{3} x_{0}^{3} \\
+30 a(000012) x_{0}^{4} x_{1} x_{2}+30 a(111102) x_{1}^{4} x_{0} x_{2}+30 a(222201) x_{2}^{4} x_{0} x_{1} \\
+60 a(000112) x_{0}^{3} x_{1}^{2} x_{2}+60 a(000221) x_{0}^{3} x_{2}^{2} x_{1}+60 a(111002) x_{1}^{3} x_{0}^{2} x_{2} \\
+60 a(111220) x_{1}^{3} x_{2}^{2} x_{0}+60 a(222001) x_{2}^{3} x_{0}^{2} x_{1}+60 a(222110) x_{2}^{3} x_{1}^{2} x_{0} \\
90 a(001122) x_{0}^{2} x_{1}^{2} x_{2}^{2} .
\end{gathered}
$$

If

$$
\widehat{F}=[F, F] / 2=Q\left(u_{0}, u_{1}, u_{2}\right)=\sum p(i j k l m n) u_{i} u_{j} u_{k} u_{l} u_{m} u_{n}
$$

then some of the coefficients of $\widehat{F}$ are written below, formulas for other coefficients can be derived with the help of permutations of indices $0,1,2$.

$$
\begin{gathered}
p(000000)=a(111111) a(222222)-10 a(111222)^{2} \\
-6 a(111112) a(222221)+15 a(111122) a(222211), \\
p(000001)=a(111112) a(222220)-a(222222) a(111110) \\
+5 a(222221) a(111102)-5 a(111122) a(222201) \\
+10 a(111222) a(222110)-10 a(222211) a(111220), \\
p(000011)=a(111122) a(222200)+a(222222) a(111100)-2 a(111102) a(222220) \\
-4 a(111222) a(222001)-4 a(222221) a(111002)
\end{gathered}
$$


$+6 a(222211) a(001122)-6 a(222110)^{2}+8 a(111220) a(222201)$,

$$
\begin{aligned}
& p(000012)=a(222220) a(111110)-a(111100) a(222221)-a(222200) a(111112) \\
& +2 a(222110) a(111220)-3 a(111102) a(222201)-6 a(111222) a(001122) \\
& +4 a(222211) a(111002)+4 a(111122) a(222001) \\
& p(000112)=a(000111) a(222221)+a(222200) a(111102) \\
& -a(000222) a(111122)-a(111100) a(222220) \\
& +3 a(111222) a(000221)+3 a(001122) a(222110)-3 a(222211) a(000112) \\
& -5 a(111220) a(222001)+2 a(222201) a(111002), \\
& p(000112)=a(000111) a(222221)+a(222200) a(111102) \\
& -a(000222) a(111122)-a(111100) a(222220) \\
& +3 a(111222) a(000221)+3 a(001122) a(222110)-3 a(222211) a(000112) \\
& -5 a(111220) a(222001)+2 a(222201) a(111002), \\
& p(000111)=a(000222) a(111222)-a(222222) a(000111) \\
& +9 a(222110) a(222001)-9 a(001122) a(222201) \\
& +3 a(222220) a(111002)+3 a(222221) a(000112) \\
& -3 a(222200) a(111220)-3 a(222211) a(000221), \\
& p(001122)=-3 a(001122)^{2} \\
& +a(000011) a(222211)+a(000022) a(111122)+a(222200) a(111100) \\
& +2 a(000112) a(222110)+2 a(000221) a(111220)+2 a(222001) a(111002) \\
& -2 a(000111) a(222201)-2 a(000222) a(111102)-2 a(111222) a(000012) \text {. }
\end{aligned}
$$

The bilinearization of these formulas generates formulas for the bimultiplication in the bialgebra of ternary sextics.

(13.2) Example. An example of a sextic idempotent (that is from $S^{6}(W)$ ). Let us consider the following sextic

$$
F=27 x_{2}^{6}-135 x_{2}^{4} x_{0} x_{1}-45 x_{2}^{2} x_{1}^{2} x_{0}^{2}+9 x_{2}\left(x_{0}^{5}+x_{1}^{5}\right)+10 x_{0}^{3} x_{1}^{3} .
$$

This (nonsingular and of genus 10) sextic is indicated by A. Wiman [31],page 533, see also formula (7) at page 638 of [9]. Wiman wrote that the sextic is invariant with respect to Valentiner's group $G_{360}$ of plane collineations (the group is isomorphic to alternating group $A_{6}$.) Using formulas (13.1), one can calculate that $\widehat{F}$ is proportional to

$$
-u_{2}^{6}+45 u_{2}^{4} u_{0} u_{1}+135 u_{2}^{2} u_{1}^{2} u_{0}^{2}-81 u_{2}\left(u_{0}^{5}+u_{1}^{5}\right)-1215 u_{0}^{3} u_{1}^{3},
$$


and that $\widehat{\widehat{F}}$ is proportional to $F$. Thus this sextic is an idempotent.

(13.3) Example of a threedimensional sextic bialgebra. After appearing the sextic in Wiman's paper [31], some further discussions of the curve were in a series of works, the works (of XIX-th century at least) are mentioned in the book [9] by Fricke-Klein. Different (but projectively equivalent) equations of Wiman's sextic are presented in [31], formula (8), page 537, in [9] one can see formulas (15), (21), (19) \& (22), (10) \& (11) at pages $625,628,633,639$ respectively.

Let us consider the following net of sextics (cf. formula (2),page 621 of [9], this net was also indicated by Wiman, see [31] formula (1), page 534).

$$
\begin{gathered}
\lambda\left(x_{0}^{6}+x_{1}^{6}+x_{2}^{6}\right)+\nu 90 x_{0}^{2} x_{1}^{2} x_{2}^{2} \\
\mu 15\left(x_{0}^{4} x_{1}^{2}+x_{0}^{4} x_{2}^{2}+x_{1}^{4} x_{0}^{2}+x_{1}^{4} x_{2}^{2}+x_{2}^{4} x_{0}^{2}+x_{2}^{4} x_{1}^{2}\right) .
\end{gathered}
$$

It is not hard to see that net (13.4) together with the corresponding net where variables are $u_{0}, u_{1}, u_{2}$ (instead of $\left.x_{0}, x_{1}, x_{2}\right)$ form a subalgebra of $\left(S^{6}(W),[],\right)$. This net contains two Wiman's sextics $C, C^{\prime}$, one corresponds to

$$
\lambda=1, \quad \mu=-\frac{5-i \sqrt{15}}{20}, \quad \nu=\frac{5+i \sqrt{15}}{30},
$$

other corresponds to conjugate numbers

$$
\lambda=1, \quad \mu=-\frac{5+i \sqrt{15}}{20}, \quad \nu=\frac{5-i \sqrt{15}}{30},
$$

(see formula (15) at page 625 of [9]). Wiman observed that the net (13.4) contains the Hessian curve of some Klein quartic (also, see (13.5) below).

(13.4) Definition. The net (13.4) or its projectively equivalent net is said to be Wiman sextic net.

(13.5) A conic description of Wiman sextic net. Let $A, B$ be two mutually apolar proper conics, $J(., .,$.$) denote the Jacobian (see (7.3), (7.4)) The net$

$$
\lambda[\widehat{A}, \widehat{B}]^{3}+\mu A B[\widehat{A}, \widehat{B}]+\nu J(A, B,[\widehat{A}, \widehat{B}])
$$

is a Wiman sextic net.

It is evident, if we use canonical coordinates from (7.8-9). Also, using the coordinates, it is not hard to see that the Hessian forms of quartics from the Wiman pencil $L_{0}(A, B)$ belong to the net. Note that the image of the pencil defines a rational cubic curve in the projective plane derived by the projectivization of the vector space of the last net.

(13.6) Example of a two-dimensional semisimple sextic subalgebra. If $A, B$ are two mutually apolar proper conics, then the pair of subspaces

$$
\begin{aligned}
& (\lambda A B[\widehat{A}, \widehat{B}]+\mu J(A, B,[\widehat{A}, \widehat{B}])) \subset S^{6}(W), \\
& (\lambda \widehat{A} \cdot \widehat{B} \cdot[A, B]+\mu J(\widehat{A}, \widehat{B},[A, B])) \quad \subset \quad S^{6}\left(W^{*}\right)
\end{aligned}
$$

is a subalgebra of $\left(S^{6}(W),[],\right)$. This subalgebra is semisimple. Indeed, using the canonical coordinates, one can see that this subalgebra is

$$
\begin{array}{ccc}
K\left(x_{0}^{6}+x_{1}^{6}+x_{2}^{6}\right)+K x_{0}^{2} x_{1}^{2} x_{2}^{2} & \subset S^{6}(W), \\
K\left(u_{0}^{6}+u_{1}^{6}+u_{2}^{6}\right)+K u_{0}^{2} u_{1}^{2} u_{2}^{2} & \subset \quad S^{6}\left(W^{*}\right),
\end{array}
$$


and that

$$
e_{1}=x_{0}^{6}+x_{1}^{6}+x_{2}^{6}, \quad e_{2}=x_{0}^{2} x_{1}^{2} x_{2}^{2}
$$

are mutually apolar idempotents (see Definitions $(1,4),(7.6)$ ).

(13.7) Example of a four-dimensional semisimple sextic subalgebra. Let $F, F^{\prime}$ be two nondegenerate ternary quadratic forms, $p, q, r, s \in W^{*}$ be four points of intersections of the conics $F=0, F^{\prime}=0$ (more precisely, four elements $p, q, r, s$ define a subset in the plane $\mathbb{P}\left(W^{*}\right)$, this subset is the intersection; also note that we can start with four points no three of which are collinear, these four points define a pencil of conics, the pencil contains a unique pair $F, F^{\prime}$ of mutually apolar conics). In terms of the bialgebra, the points are four independent solutions of the system

$$
<x^{2}, F>=0, \quad<x^{2}, F^{\prime}>=0 .
$$

Let linear forms $a, b, c, d$ define tangents to $F$ at points $p, q, r, s$ respectively. The linear forms can be defined up to proportionality by the following conditions.

$$
\begin{aligned}
& <a, p>=0, \quad<a^{2}, \widehat{F}>=0, \\
& <b, q>=0, \quad<b^{2}, \widehat{F}>=0, \\
& <c, r>=0, \quad<c^{2}, \widehat{F}>=0, \\
& <d, s>=0, \quad<d^{2}, \widehat{F}>=0 .
\end{aligned}
$$

Similarly, let linear forms $a^{\prime}, b^{\prime}, c^{\prime}, d^{\prime}$ define tangents to $F^{\prime}$ at points $p, q, r, s$ respectively. Further, let $k, l, m, n$ be four bitangents for the pair of conics, $u, v, w, z, u^{\prime}, v^{\prime}, w^{\prime}$, $z^{\prime} \in W^{*}$ be eight points such that in the projective plane $\mathbb{P}\left(W^{*}\right)$, we have (using some inaccurate notations)

$$
\begin{gathered}
\{u\}=F \cap k,\{v\}=F \cap l,\{w\}=F \cap m,\{z\}=F \cap n, \\
\left\{u^{\prime}\right\}=F^{\prime} \cap k,\left\{v^{\prime}\right\}=F^{\prime} \cap l,\left\{w^{\prime}\right\}=F^{\prime} \cap m,\left\{z^{\prime}\right\}=F^{\prime} \cap n .
\end{gathered}
$$

Let

$$
\begin{aligned}
& U=U\left(F, F^{\prime}\right)=\left(K F a^{\prime} b^{\prime} c^{\prime} d^{\prime}+K F^{\prime} a b c d\right. \\
& \left.+K\left[\widehat{F}, \widehat{F^{\prime}}\right]^{3}+K F F^{\prime}\left[\widehat{F}, \widehat{F^{\prime}}\right]\right) \subset \quad S^{6}(W), \\
& V=V\left(F, F^{\prime}\right)=\left(\widehat{F} u^{\prime} v^{\prime} w^{\prime} z^{\prime}+K \widehat{F^{\prime}} u v w z\right. \\
& \left.+K\left[F, F^{\prime}\right]^{3}+K \widehat{F} \widehat{F^{\prime}}\left[F, F^{\prime}\right]\right) \subset \quad S^{6}\left(W^{*}\right) .
\end{aligned}
$$

Theorem. The pair of subspaces $(U, V)$ is a fourdimensional semisimple subalgebra of $\left.S^{6}(W),[],\right)$.

Proof. Let us use the canonical coordinates (7.9), (7.8), (also see proof of Theorem (7.15)). The above mentioned objects have the following coordinate description.

$$
\begin{gathered}
F=x_{0}^{2}+\omega x_{1}^{2}+\omega^{2} x_{2}^{2}, \quad F^{\prime}=x_{0}^{2}+\omega^{2} x_{1}^{2}+\omega x_{2}^{2}, \\
p=u_{0}-u_{1}-u_{2}, \quad q=-u_{0}+u_{1}-u_{2}, \\
r=-u_{0}-u_{1}+u_{2}, \quad s=u_{0}+u_{1}+u_{2}, \\
u=-u_{0}+\omega^{2} u_{1}+\omega u_{2}, \quad v=u_{0}-\omega^{2} u_{1}+\omega u_{2}, \\
w=u_{0}+\omega^{2} u_{1}-\omega u_{2}, \quad z=u_{0}+\omega^{2} u_{1}+\omega u_{2},
\end{gathered}
$$




$$
\begin{gathered}
u^{\prime}=-u_{0}+\omega u_{1}+\omega^{2} u_{2}, \quad v^{\prime}=u_{0}-\omega u_{1}+\omega^{2} u_{2}, \\
w^{\prime}=u_{0}+\omega u_{1}-\omega^{2} u_{2}, \quad z^{\prime}=u_{0}+\omega u_{1}+\omega^{2} u_{2}, \\
k=x_{0}-x_{1}-x_{2}, \quad l=-x_{0}+x_{1}-x_{2}, \\
m=-x_{0}-x_{1}+x_{2}, \quad n=x_{0}+x_{1}+x_{2}, \\
a=-x_{0}+\omega x_{1}+\omega^{2} x_{2}, \quad a^{\prime}=-x_{0}+\omega^{2} x_{1}+\omega x_{2}, \\
b=x_{0}-\omega x_{1}+\omega^{2} x_{2}, \quad b^{\prime}=x_{0}-\omega^{2} x_{1}+\omega x_{2}, \\
c=x_{0}+\omega x_{1}-\omega^{2} x_{2}, \quad c^{\prime}=x_{0}+\omega^{2}-x_{1} \omega x_{2}, \\
d=x_{0}+\omega x_{1}+\omega^{2} x_{2}, \quad d^{\prime}=x_{0}+\omega^{2} x_{1}+\omega x_{2}, \\
U=\left(K\left(x_{0}^{6}+x_{1}^{6}+x_{2}^{6}\right)+K x_{0}^{2} x_{1}^{2} x_{2}^{2}\right. \\
+K\left(x_{0}^{4} x_{1}^{2}+x_{1}^{4} x_{2}^{2}+x_{2}^{4} x_{0}^{2}\right) \\
\left.+K\left(x_{0}^{4} x_{2}^{2}+x_{2}^{4} x_{1}^{2}+x_{1}^{4} x_{0}^{2}\right)\right), \\
V=\left(K\left(u_{0}^{6}+u_{1}^{6}+u_{2}^{6}\right)+K u_{0}^{2} u_{1}^{2} u_{2}^{2}\right. \\
+K\left(u_{0}^{4} u_{1}^{2}+u_{1}^{4} u_{2}^{2}+u_{2}^{4} u_{0}^{2}\right) \\
\left.+K\left(u_{0}^{4} u_{2}^{2}+u_{2}^{4} u_{1}^{2}+u_{1}^{4} u_{0}^{2}\right)\right)
\end{gathered}
$$

It is easy to see (with the help of (10.12), (10.13) or (13.1)) that elements

$$
\begin{gathered}
e_{1}=x_{0}^{6}+x_{1}^{6}+x_{2}^{6}, \quad e_{2}=x_{0}^{2} x_{1}^{2} x_{2}^{2}, \\
e_{3}=x_{0}^{4} x_{1}^{2}+x_{1}^{4} x_{2}^{2}+x_{2}^{4} x_{0}^{2}, \quad e_{4}=x_{0}^{4} x_{2}^{2}+x_{2}^{4} x_{1}^{2}+x_{1}^{4} x_{0}^{2},
\end{gathered}
$$

are idempotents and mutually apolar.

The theorem is proved.

(13.8) Remark. If $F, F^{\prime}$ are mutually apolar proper quadratic forms, then there is a linear dependence between the following five ternary sextics.

$$
\begin{aligned}
& F a^{\prime} b^{\prime} c^{\prime} d^{\prime}, F^{\prime} a b c d,\left[\widehat{F}, \widehat{F^{\prime}}\right]^{3}, \\
& F F^{\prime}\left[\widehat{F}, \widehat{F^{\prime}}\right], J\left(F, F^{\prime},\left[\widehat{F}, \widehat{F^{\prime}}\right]\right) .
\end{aligned}
$$

For example, if the quadratic forms are written with the help of the canonical coordinates, as in the proof, then

$$
8\left(F a^{\prime} b^{\prime} c^{\prime} d^{\prime}+F^{\prime} a b c d\right)-24\left[\widehat{F}, \widehat{F^{\prime}}\right]^{3}+8 F F^{\prime}\left[\widehat{F}, \widehat{F^{\prime}}\right]+9 J\left(F, F^{\prime},\left[\widehat{F}, \widehat{F^{\prime}}\right]\right)=0 .
$$

Therefore the space $U\left(F, F^{\prime}\right)$ contains the pencil from (13.6), Wiman's net from (13.5) or (13.2) and all the above mentioned sextics.

(13.9) Definition of the Edge pencil. The Edge pencil is

$$
E\left(F, F^{\prime}\right)=\left(K F a^{\prime} b^{\prime} c^{\prime} d^{\prime}+K F^{\prime} a b c d\right) \subset U\left(F, F^{\prime}\right) .
$$

The pencil was studied by W. L. Edge in [7] . The following special base $\{W, P\}$ of the pencil was also indicated by Edge. If $F, F^{\prime}, a, b, c, d, a^{\prime}, b^{\prime}, c^{\prime}, d^{\prime}$ are presented with the help of canonical coordinates as in the proof, then

$$
\begin{gathered}
W=F a^{\prime} b^{\prime} c^{\prime} d^{\prime}+F^{\prime} a b c d=x_{0}^{6}+x_{1}^{6}+x_{2}^{6} \\
+\left(x_{0}^{2}+x_{1}^{2}+x_{2}^{2}\right)\left(x_{0}^{4}+x_{1}^{4}+x_{2}^{4}\right)-12 x_{0}^{2} x_{1}^{2} x_{2}^{2},
\end{gathered}
$$




$$
P=\left(\omega^{2}-\omega\right)^{-1}\left(F a^{\prime} b^{\prime} c^{\prime} d^{\prime}-F^{\prime} a b c d\right)=\left(x_{1}^{2}-x_{2}^{2}\right)\left(x_{2}^{2}-x_{0}^{2}\right)\left(x_{0}^{2}-x_{1}^{2}\right) .
$$

(13.10) Remark. A sextic projectively equivalent to $W=0$ was indicated by Wiman [32], page 208. The sextic $W$ admits a group $G_{120}$ of 120 Cremona selftransformations (of which 24 are projective transformations). Another two remarkable sextics $R, R^{\prime}$ from the Edge pencil are

$$
W+\lambda P=0
$$

where

$$
\lambda=5 \sqrt{5} \text { or } \lambda=-5 \sqrt{5} .
$$

Both the curves are ten-nodal rational sextics. Four of the nodes are $p, q, r, s$ (these points are singular for all the curves of the Edge pencil. If $\lambda=5 \sqrt{5}$ (and the canonical coordinates are used), then corresponding curve $R$ has nodes at the points

$$
\begin{array}{ccc}
(0: \tau: 1), & (\tau: 1: 0), & (1: 0: \tau), \\
(0:-\tau: 1), & (-\tau: 1: 0), & (1: 0:-\tau),
\end{array}
$$

where

$$
\tau=(1+\sqrt{5}) / 2
$$

See [7] for details.

\section{REFERENCES}

[1] H. F. BAKER, An Introduction to Plane Geometry, Cambridge, 1943.

[2] F. Brioschi, Sopra una classe di curve del $4^{\circ}$ ordine, Atti della Reale Accademia dei Lincei, serie terza, 8 (1883-1884), Roma, pp. 164-168.

[3] W. Burnside, On the Hessian configuration and its connection with the group of 360 plane collineations, Proceedings of the London Mathematical Society (2), 4 (1907), pp. 54-71.

[4] E. Ciani, Il theorema sopra il covariante $S$ delle quartice piani, Rendiconti Circolo Methematico del Palermo, 14 (1900), pp. 16-21.

[5] I. V. Dolgachev and V. Kanev, Polar covariants of plane cubics and quartics, Advances in Mathematics, 98:2 (1993), pp. 216-301.

[6] W. L. EDGE, Determinantal representation of $x^{4}+y^{4}+z^{4}$, Proceedings of the Cambridge Philosophical Society, 34 (1938), pp. 6-21.

[7] W. L. Edge, A pencil of four-nodal plane sextics, Mathematical Proceedings of the Cambridge Philosophical Society, 89 (1981), pp. 413-421.

[8] L. R. Ford, Automorphic Functions, Chelsea, 1929, 1951.

[9] R. Fricke AND F. Klein, Vorlesungen Über Automorphen Funktionen, Band II, Teubner, Leipzig, 1912.

[10] von GaLl, Das vollständige Formensystem einer binären Form achter Ordnung, Mathematische Annalen, 17 (1880), pp. 31-51.

[11] F. Gerbaldi, Sui gruppi di sei coniche in involuzione, Torino Atti, 17 (1882), pp. 358-371, 566-580.

[12] F. Gerbaldi, Sul gruppo semplice di 360 collineazione piane, Rendiconti del circolo matematico di Palermo; I, 12 (1898), pp. 23-94; II, 13 (1899). pp. 161-169; III, 14 (1900), pp. 66-114; IV, 16 (1902), pp. 129-154.

[13] F. Gerbaldi, Sul gruppo semplice di 360 collineazione piane, Mathematische Annalen, 50 (1900), pp. 473-476.

[14] F. Gerbaldi, Sul gruppo semplice di 360 collineazione piane, in Verhandlungen der ersten internationalen Mathematiker-Kongresses in Zürich vom 9. bis 11. August 1897, Leipzig, 1898, pp. 242-246.

[15] P. Gordan, Über das Formensystem der binären Formen, Teubner, Leipzig, 1875. 
[16] P. Gordan, Die partielle Differentiale Gleichungen der Valentinerproblem, Mathematische Annalen, 61 (1906), pp. 453-526.

[17] W. V. D. Hodge And D. Pedoe, Methods of Algebraic Geometry II, Cambridge, 1952.

[18] F. KLEIN, Über die Transformation siebenter Ordnung der elliptischer Funktionen, Mathematische Annalen, 14 (1878-1879), pp. 428-471; Gesammelte Abhandlungen III, Springer-Verlag, pp. 90-136.

[19] F. KLEIN, Allgemeine Gleichungen fünften und sechsten Grades, Journal für die reine und angewandte Mathematik, 129 (1905), pp. 151-174; Mathematische Annalen, 61 (1905), pp. 50-71; in Gesammelte Abhandlungen III, Springer-Verlag, 1923,1973, pp. 481-504.

[20] F. KLEIN, Elementarmathematik von höheren Standpunkt aus, Springer, Berlin, 1933, 1968.

[21] G. Kohn And G. Loria, Spezielle ebene algebraische Kurven, in Encyklopädie der mathematischen Wissenschaften III-C, Heft 5, Teubner, Leipzig, 1898-1904.

[22] M. PASH, Ueber eine Invariante der trilinearen ternären Formen, Mathematische Annalen, 52 (1899), pp. 127-129.

[23] R. E. Rodríguez and V. González-Agullera, Fermat's Quartic Curve, Klein's Curve and the Tetrahedron, Contemporary mathematics, 201 (1997), pp. 43-62.

[24] G. Salmon, A Treatise on Conic Sections, London, 1878.

[25] G. Salmon, A Treatise on the Higher Plane Curves, Third edition, Dublin, 1879.

[26] J. G. Semple and G. T. Kneebone, Algebraic Projective Geometry, Oxford, 1952.

[27] E. Steinitz, Konfigurationen der projektiven Geometrie, in Encyklopädie der mathematischen Wissenschaften Band III-1, Heft 4, Teubner, Leipzig, 1898-1904, pp. 481-516.

[28] H. Valentiner, De endelige Transformations-Gruppers Theori, Videnskabernes Selkabs Skrifter, 6. Raekke, 5 (1899), Kjób, pp. 64-135.

[29] H. WeBer, Lehrbuch der Algebra II, Braunschweig, 1896.

[30] W. G. Welchman, Introduction to Algebraic Geometry, At the University press, Cambridge, 1950.

[31] A. Wiman, Eine einfache Gruppe von 360 ebenen Collineationen, Mathematische Annalen, 47 (1896), pp. 531-556.

[32] A. Wiman, Zur Theorie der endlichen Gruppen von Gruppen von birationalen Transformationen der Ebene, Mathematische Annalen, 48 (1897), pp. 193-240.

[33] A. Wiman, Endliche Gruppen linearen Substitutionen, in Encyklopädie der mathematischen Wissenschaften BandI, Teubner, Leipzig, 1898-1904, pp. 522-554.

[34] S. Grass, Solving the sextic by iteration: A study in complex geometry an dynamics, Experimental mathematics, 8:3 (1898-1999), pp. 209-240.

[35] J. Dixmier, Enveloping Algebras, American Mathematical Society, 1977, 1996. 